JOURNAL OF

SYMPLECTIC GEOMETRY

Volume 11, Number 4, 603-643, 2013

\title{
DEGENERATION OF KÄHLER STRUCTURES AND HALF-FORM QUANTIZATION OF TORIC VARIETIES
}

\author{
William D. Kirwin, José M. Mourão and João P. Nunes
}

We study the half-form Kähler quantization of a smooth symplectic toric manifold $(X, \omega)$, such that $[\omega / 2 \pi]-c_{1}(X) / 2 \in H^{2}(X, \mathbb{Z})$ and is non-negative. We define the half-form corrected quantization of $(X, \omega)$ to be given by holomorphic sections of a certain Hermitian line bundle $L \rightarrow X$ with Chern class $[\omega / 2 \pi]-c_{1}(X) / 2$. These sections then correspond to integral points of a "corrected" polytope $P_{L}$ with integral vertices. For a suitably translated moment polytope $P_{X}$ for $(X, \omega)$, we have that $P_{L} \subset P_{X}$ is obtained from $P_{X}$ by a one-half inward-pointing normal shift along the boundary.

We use our results on the half-form corrected Kähler quantization to motivate a definition of half-form corrected quantization in the singular real toric polarization. Using families of complex structures studied in [BFMN11], which include the degeneration of Kähler polarizations to the vertical polarization, we show that, under this degeneration, the half-form corrected $L^{2}$-normalized monomial holomorphic sections converge to Dirac-delta-distributional sections supported on the fibers over the integral points of $P_{L}$, which correspond to corrected BohrSommerfeld fibers. This result and the limit of the corrected connection, with curvature singularities along the boundary of $P_{X}$, justifies the direct definition we give for the corrected quantization in the singular real toric polarization. We show that the space of quantum states for this definition coincides with the space obtained via degeneration of the Kähler quantization.

We also show that the BKS pairing between Kähler polarizations is not unitary in general. On the other hand, the unitary connection induced by this pairing is flat.

\section{Contents}

\section{Introduction}


2.1. Complex line bundles 608

2.2. Toric manifolds $\quad 610$

2.2.1. The symplectic structure of $X \quad 610$

2.2.2. The Kähler structure of $X \quad 612$

2.2.3. Line bundles and sections $\quad 614$

2.2.4. The canonical bundle $\quad 617$

3. Half-form corrected Kähler quantization 620

$\begin{array}{ll}\text { 3.1. Motivation } & 620\end{array}$

3.2. Corrected quantization $\quad 622$

4. Half-form corrected quantization in the singular real toric polarization $\quad 626$

4.1. Distributional sections $\quad 626$

4.2. Quantization in the (singular) real polarization $\quad 627$

4.3. Degeneration to the real polarization 631

5. The BKS pairing $\quad 636$

5.1. The BKS half-form pairing $\quad 636$

5.2. Unitarity 637

5.3. Quantum connection $\quad 639$

6. Appendix: The square root of $K \quad 640$

References $\quad 641$

\section{Introduction}

Ever since Śnyaticki proposed cohomological wave functions to construct the quantum Hilbert space corresponding to geometric quantization in real

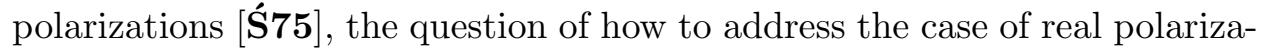
tions with singular fibers has resisted full treatment. In [Ham07], Hamilton proposed the extension of Śnyaticki's definition to the case with singular fibers by also considering the higher cohomology of the same sheaf of polarized smooth sections of the prequantization bundle. His results show, however, that the formalism will have to be modified in order to obtain the expected quantization even in the case of the harmonic oscillator. Indeed, for singularities of elliptic type (like in the case of toric varieties) Hamilton obtains states corresponding only to non-singular Bohr-Sommerfeld leaves. In the toric case, these correspond to interior integral points of the moment polytope. If one doesn't take into account the half-form correction, however, one expects the quantization to include all states corresponding to the integral points of the polytope, including those on the boundary. Only in this way, for the compact case, does one get the same dimension of the space of quantum states as for the holomorphic polarizations. 
In [BFMN11], a solution of this problem was proposed within the context of toric varieties without the half-form correction. The real polarized sections are defined directly as distributional solutions of the equations of covariant constancy and can also be obtained by degenerating appropriately normalized Kähler polarized sections. These normalized holomorphic sections converge, under the degeneration, to Dirac-delta-distributional sections supported on the Bohr-Sommerfeld fibers which correspond to integral points of the moment polytope, including the ones on the boundary. The corresponding Bohr-Sommerfeld orbits are increasingly singular (lower dimensional) as the codimension of the face of the polytope on which they are increases.

On the other hand, one would expect these quantum states not to be present in a quantization in the real toric polarization correctly reproducing the "vacuum energy shift" of the harmonic oscillator. We show that this expected behavior of the quantum states is precisely achieved by our definition of the half-form corrected Kähler quantizations deforming continuously to the real polarization.

An immediate obstacle to defining the half-form quantization in a Kähler polarization is the fact that the canonical bundle $K_{X}$ of a toric variety may not admit a square root, for instance for $\mathbb{C P}^{2 n}$. (See the appendix for a discussion of the existence of $\sqrt{K_{X}}$ in terms of the fan of $X$.) In Section 3, we consider Kähler quantization of a compact toric manifold $X$ with symplectic structure $\omega$ such that $\frac{[\omega]}{2 \pi}-\frac{c_{1}(X)}{2} \in H^{2}(X, \mathbb{Z})$ and is non-negative. (This integrality condition has also been proposed in [C78].) In the case when $c_{1}(X)$ is even, so that $K_{X}$ admits a square root, one is then reduced to the usual setting for half-form quantization. Let $L \rightarrow X$ be an Hermitian line bundle with connection of curvature given by $-\mathrm{i} \omega+\frac{\mathrm{i}}{2} \rho$, where $\rho$ is the Ricci form for the Kähler metric on $X$, so that $[\rho / 2 \pi] \in c_{1}(X)$. When a $\sqrt{K_{X}}$ exists, this corresponds to taking the usual prequantum connection plus one-half the Chern-Levi-Civita connection on $K_{X}$, which gives a connection on $\sqrt{K_{X}}$.

The condition $\frac{[\omega]}{2 \pi}-\frac{c_{1}(X)}{2} \in H^{2}(X, \mathbb{Z})$ allows us to choose the moment polytope (see equations (3.6) and (3.7) in Section 3.2), $P_{X}=\mu(X)$,

$$
P_{X}=\left\{x \in \mathbb{R}^{n}: \ell_{j}(x)=\nu_{j} \cdot x+\lambda_{j} \geq 0, \quad j=1, \ldots, r\right\},
$$

with all $\lambda_{j}$ 's half-integral

$$
\lambda_{j} \in \frac{1}{2}+\mathbb{Z}, \quad j=1 \ldots, r,
$$

where $x$ are action coordinates and $\nu_{j}$ is the primitive inward pointing normal vector to the $j$ th facet. With this choice, there are no integral points in the boundary of $P_{X}$ and to all integral points inside $P_{X}$ there will correspond Kähler polarized states, that is holomorphic sections of 
$L$. Unlike Hamilton's case however, the integral points start at lattice distance 1/2 (rather than 1), from every facet (see the figures in Remarks 3.7 and 3.8.)

As in the case without the half-form correction, these polarized states will converge, as the polarizations degenerate to the toric real polarization, to delta distributions supported on the corresponding non-singular orbits. The degeneration of the equations for polarized sections (see Section 4.2) is also consistent with the degeneration of the polarized states as the Ricci connection term i $d \theta_{v}^{j}$ in (4.2) corresponds to a connection with curvature supported on the inverse image of the $j$ th facet by the moment map. From the point of view of the real polarization these singular connections are responsible for the vacuum energy shifts (which correspond to shifted BohrSommerfeld conditions) as they prevent the existence of covariantly constant sections supported on the boundary. Quantization in the real singular toric polarization is then defined directly in terms of this limit connection. This provides an approach for defining half-form corrected quantization in real singular polarizations. By finding the type of singularities of the half-form corrected limit connection, one finds corrected equations for the real polarized sections. In the toric case, this direct approach for the definition of the half-form corrected quantization in the singular toric real polarization gives the same results as the degeneration of Kähler polarizations (Theorems 4.7 and 4.15).

In [BFMN11], the convergence to delta-distributions was achieved by taking $L^{1}$-normalized sections. In the present paper, however, the half-form correction ensures the nice behavior of the $L^{2}$-normalized sections in the limit of degenerating complex structure. This is in agreement with other examples such as finite-dimensional vector spaces [KW06] and abelian varieties [BMN10].

One of the primary motivations for including the half-form correction is that it allows for a canonical pairing between quantizations associated to different complex structures. This pairing is known as the Blattner-KonstantSternberg (BKS) pairing. The BKS pairing between quantizations associated to two Kähler complex structures is non-degenerate, and hence (since the Kähler quantizations of a compact toric manifold are finite dimensional) induces an isomorphism between them. One does not, although, expect in general that the BKS pairing provides a unitary identification of quantizations associated to different complex structures. In several common cases, for example for symplectic vector spaces equipped with translation invariant Kähler structures, and for complex Lie groups equipped with certain families of Kähkler structures (which include the canonical Kähler structure), the BKS pairing is unitary (see [Hal02, KW06, FMMN05, FMMN06]). In a few other cases, the BKS pairing is known to be not unitary, for example for $T^{*} S^{2}$ [Raw79]. In most cases, it is not known whether the BKS pairing is 
unitary, and conditions for unitarity do not yet seem to be well understood. We show that the BKS pairing between half-form corrected quantizations of compact toric varieties is not unitary in general.

We will also consider another method for comparing quantizations associated to different complex structures. Namely, one can construct a (finiterank) Hilbert bundle over the space of toric complex structures on $X$ with the fiber at a point being the quantum Hilbert space associated to that complex structure. When the half-form correction is not included, the quantum Hilbert bundle is a subbundle of a trivial bundle, and hence carries a canonical connection obtained by orthogonal projection of the trivial connection. This connection is called the quantum connection. It was first introduced and studied by Axelrod, della Pietra and Witten in [APW91] and, from a slightly different point of view, by Hitchin in [Hit90]. See also [AGL07] for a treatment that includes the half-form correction.

For linear complex structures on a symplectic vector space, the quantum connection turns out to be projectively flat, which means that up to a constant, one may identify all Kähler quantizations at once. To extend the connection to the boundary of the space of complex structures, and thus study their degenerations and relate real quantizations to Kähler quantizations, one must introduce the half-form correction; parallel transport of the resulting corrected quantum connection, still in the case of linear complex structures, was studied by the first author and $\mathrm{Wu}$ in [KW06], where it was found that parallel transport along geodesics with internal endpoints is just rescaled Bergman projection, while transport along geodesics with one or two endpoints on the boundary yields the well-known Segal-Bargmann and Fourier transforms, respectively. These results were recently extended by $\mathrm{Wu}$ to the case of linear quantization of fermions [Wu10]. In the usual (bosonic) case, one may then quotient by the action of $\mathbb{Z}^{2 n}$, as done by Baier and the second two authors in [BMN10], to study degenerations of complex structures on abelian varieties at the level of $\vartheta$-functions. In a different direction, in [FMMN05] and [FMMN06], Florentino, Matias and the second two authors studied the corrected quantum connection on a one-dimensional family of complex structures on the complexification of a compact Lie group which degenerates to the vertical polarization of the cotangent bundle of the underlying real Lie group; here, again, parallel transport with respect to the quantum connection yields the (generalized) Segal-Bargmann-Hall transform. In related work, Lempert and Szőke have recently studied the bundle of quantizations associated to a family of adapted-type complex structures on Grauert tubes of compact, real-analytic Riemannian manifolds [LS10], although they use a Chern-type connection rather than the BKS construction considered here.

In Section 5.3, we show that the quantum connection on the quantum Hilbert bundle induced by the BKS pairing is flat, so that the quantizations 
associated to different torus-invariant complex structures can be canonically identified.

\section{Preliminaries}

2.1. Complex line bundles. We begin with some facts about complex line bundles. Let $E \rightarrow X$ be a complex line bundle on a manifold $X$ and let $E_{0}=E \backslash\{$ zerosection $\}$ be its frame bundle. The isomorphism

$$
\begin{aligned}
(|\cdot|, \arg ): \mathbb{C}^{*} & \cong \mathbb{R}^{+} \times U(1) \\
c & \mapsto\left(|c|, \frac{c}{|c|}\right),
\end{aligned}
$$

defines a canonical isomorphism ([Wei04], p. 6)

$$
E \cong|E| \otimes E^{U(1)}
$$

where the complex line bundles $|E|, E^{U(1)}$ are associated to the principal $\mathbb{C}^{*}$-bundle $E_{0}$, via the homomorphisms $\mathbb{C}^{*} \ni c \mapsto|c| \in \mathbb{R}^{+}$and $\mathbb{C}^{*} \ni c \mapsto$ $\arg (c)=\frac{c}{|c|} \in U(1)$, respectively. Following [Wei04] we call the line bundle $E^{U(1)}$ the unitarization of $E$.

This isomorphism is given explicitly by

$$
E_{p} \ni l \mapsto \begin{cases}|l| \otimes l^{U(1)}, & l \neq 0, \\ 0, & l=0,\end{cases}
$$

where $p \in X,|l|=[(l, 1)]_{|\cdot|}=\left[\left(l c^{-1},|c|\right)\right]_{|\cdot|} \in|E|=E_{0} \times_{\left(\mathbb{C}^{*},|\cdot|\right)} \mathbb{C}$ and $l^{U(1)}=$ $[(l, 1)]_{\mathrm{arg}}=\left[\left(l c^{-1}, \frac{c}{|c|}\right)\right]_{\mathrm{arg}} \in E^{u(1)}=E_{0} \times\left(\mathbb{C}^{*}, \arg \right) \mathbb{C}, c \in \mathbb{C}^{*}$.

For simplicity, we will identify $E$ with $|E| \otimes E^{U(1)}$ and write $0 \neq l=$ $|l| \otimes l^{U(1)}=|l| l^{U(1)}$ and thus, also, $l^{U(1)}=\frac{l}{|l|}$.

Let $\left\{g_{\alpha \beta}\right\}$ be the transition functions for $E$ associated to local trivializations for some open cover $\left\{U_{\alpha}\right\}$ of $X$. Then, for the same open cover $\left\{U_{\alpha}\right\}$, the complex line bundle $E^{U(1)}$ has $U(1)$-valued transition functions $\left\{g_{\alpha \beta} /\left|g_{\alpha \beta}\right|\right\}$, and the complex line bundle $|E|$ has $\mathbb{R}^{+}$-valued transition functions $\left\{\left|g_{\alpha \beta}\right|\right\}$.

This decomposition of $E=|E| \otimes E^{U(1)}$ induces an associated splitting of connections. Let $\nabla$ be a connection on $E$ with connection form $\Theta$ associated to a local trivializing section $s, \nabla s=\Theta s$. Since, at the level of Lie algebras, the isomorphism $\mathbb{C}^{*} \cong \mathbb{R}^{+} \times U(1)$ gives $\mathbb{C} \cong \mathbb{R} \oplus \mathrm{i} \mathbb{R}$, we have $\nabla=\nabla^{|E|}+$ $\nabla^{E^{U(1)}}$ where $\Theta^{|E|}=\operatorname{Re} \Theta$ and $\Theta^{E^{U(1)}}=\mathrm{i} \operatorname{Im} \Theta$ are the connection forms for $|E|, E^{U(1)}$ associated to the local trivializing sections $|s|, s^{U(1)}$, respectively:

$$
\begin{aligned}
\nabla^{|E|}|s| & =\operatorname{Re} \Theta|s|, \\
\nabla^{E^{U(1)}} s^{U(1)} & =\mathrm{i} \operatorname{Im} \Theta s^{U(1)} .
\end{aligned}
$$


Now let $E$ have an Hermitian structure $h$. Then $|E|$ has a global trivializing section $\mu_{h}$ defined as follows. Let $s$ be any local trivializing section of $E$ over an open set $U \subset X$. Over $U$, define

$$
\mu_{h}=\frac{|s|}{\sqrt{h(s, s)}} .
$$

Note that, since $h$ is an Hermitian structure, $\mu_{h}$ is independent of the choice of the local trivializing section $s$ and therefore extends to a global trivializing section of $|E|$.

Let $\Gamma(E)$ denote the space of smooth sections of $E$. A connection $\nabla$ on $(E, h) \rightarrow X$ is said to be compatible with the Hermitian structure if for any section $s \in \Gamma(E)$, one has $d h(s, s)=h(\nabla s, s)+h(s, \nabla s)$. Let $\|s\|^{2}=$ $h(s, s)$. This property is equivalent to $d\|s\|=\operatorname{Re} \Theta\|s\|$ which is, in turn, equivalent to

$$
\nabla^{|E|} \mu_{h}=0
$$

Remark 2.1. The above isomorphism of $|E|$ with the trivial bundle also defines, since $E=|E| \otimes E^{U(1)}$, an isomorphism of $E$ with $E^{U(1)}$ given by $l \mapsto l \frac{\sqrt{h(l, l)}}{|l|}, l \in E$.

Remark 2.2. If $X$ is a complex manifold and if $E$ has an holomorphic structure, then given a global non-zero meromorphic section of $E, s$, one has $\mu_{h}=\frac{|s|}{\sqrt{h(s, s)}}$ away from the divisor of $s$. This expression extends uniquely to $\mu_{h}$ on the whole of $X$.

Any line bundle $E^{U(1)}$ has a canonical Hermitian structure defined by $\widetilde{h}\left(z l^{U(1)}, z^{\prime} l^{U(1)}\right)=z \bar{z}^{\prime}$. This Hermitian structure is independent of the choice of representative $l$ and is therefore well defined.

Remark 2.3. An Hermitian line bundle $(E, h) \rightarrow X$ can then be decomposed into smooth Hermitian line bundles $(E, h)=(|E|, \widehat{h}) \otimes\left(E^{U(1)}, \widetilde{h}\right)$. The Hermitian structure on $|E|$ is defined by $\widehat{h}\left(z|l|, z^{\prime}|l|\right)=h(l, l) z \bar{z}^{\prime}$, so that

$$
h\left(z l, z^{\prime} l\right)=z \bar{z}^{\prime} \widehat{h}(|l|,|l|) \cdot \widetilde{h}\left(l^{U(1)}, l^{U(1)}\right)=z \bar{z}^{\prime} \widehat{h}(|l|,|l|), \quad z, z^{\prime} \in \mathbb{C}, l \in E .
$$

Remark 2.4. Note that under the isomorphism $|E| \simeq X \times \mathbb{C}$ defined by $\mu_{h}$, the Hermitian form $\widehat{h}$ becomes the standard Hermitian product on $\mathbb{C}$.

We recall the following standard results (see, for instance, Proposition 4.2.14 in [Huy05]):

Lemma 2.5. If $(E, h) \rightarrow X$ is a complex Hermitian vector bundle, then there exists a compatible connection $\nabla$. Moreover, $\nabla^{\prime}$ is another compatible connection if and only if there exists a (global) real-valued 1 -form $\beta$ such that $\nabla^{\prime}=\nabla+\mathrm{i} \beta$. 
If $E \rightarrow X$ is a holomorphic line bundle, then $\bar{\partial}$ is a well-defined operator on $\Gamma(E)$. A connection $\nabla$ on $E$ is said to be compatible with the holomorphic structure if $\nabla^{0,1}=\bar{\partial}$.

Lemma 2.6. Let $(E, h) \rightarrow X$ be a Hermitian holomorphic line bundle. There exists a unique connection $\nabla$, called the Chern connection, which is compatible with both the Hermitian structure $h$ and the holomorphic structure of $E$. Moreover, if $\left\{U_{\alpha}, s_{\alpha}\right\}$ is a holomorphic trivialization of $E$, then $\nabla s_{\alpha}=\left(\partial \log h\left(s_{\alpha}, s_{\alpha}\right)\right) s_{\alpha}$.

Then, in the holomorphic local trivialization $\left\{U_{\alpha}, s_{\alpha}\right\}$, we have

$$
F_{\nabla}=d\left(\partial \log h\left(s_{\alpha}, s_{\alpha}\right)\right)=-\partial \bar{\partial} \log h\left(s_{\alpha}, s_{\alpha}\right)
$$

that is, $-\log h\left(s_{\alpha}, s_{\alpha}\right)$ is a local potential for the curvature 2-form and, on the open set $U_{\alpha}$,

$$
\left[\mathrm{i} \partial \bar{\partial}\left(-\log h\left(s_{\alpha}, s_{\alpha}\right)\right)\right] \in 2 \pi \cdot c_{1}(E) .
$$

The induced connections on $|E|$ and $E^{U(1)}$ are then given by

$$
\begin{aligned}
\nabla^{|E|}|s| & =\frac{1}{2} d \log h(s, s)|s|, \\
\nabla^{E^{U(1)}} s^{U(1)} & =\frac{1}{2}(\partial \log h(s, s)-\bar{\partial} \log h(s, s)) s^{U(1)} .
\end{aligned}
$$

If $X$ is Kähler with integral symplectic form $\omega$, and $L$ is an Hermitian holomorphic line bundle with the curvature of the Chern connection given by $-\mathrm{i} \omega$, then in a local holomorphic trivialization one has that $\kappa=-\log h(s, s)$ is a local Kähler potential.

2.2. Toric manifolds. Let $(X, \omega)$ be a compact smooth symplectic toric manifold with symplectic form $\omega$, moment map $\mu: X \rightarrow \operatorname{Lie}\left(\mathbb{T}^{n}\right)^{*} \simeq \mathbb{R}^{n}$ and moment polytope $P_{X}=\mu(X)$ with associated fan $\Sigma$. The Kähler structure of $X$, which connects the symplectomorphism class of $X$ determined by $P_{X}$ to the biholomorphism class of $X$ determined by $\Sigma$, is fixed by choosing a so-called symplectic potential. We will find both descriptions, as well as the relation between them, essential for our work, and so we describe them briefly here (although we refer the interested reader to [Gui94, Abr03, CLS11, DP09] for details).

2.2.1. The symplectic structure of $\boldsymbol{X}$. Let $\check{P}_{X}$ denote the interior of the moment polytope $P_{X}$. On $\check{X}=\mu^{-1}\left(\check{P}_{X}\right) \cong \check{P}_{X} \times \mathbb{T}^{n}$ consider action-angle coordinates $(x, \theta)$, so that $\mu(x, \theta)=x={ }^{t}\left(x^{1}, \ldots, x^{n}\right)$. The symplectic form $\omega$ in this coordinate chart is simply

$$
\left.\omega\right|_{\mu^{-1}\left(\check{P}_{X}\right)}=\sum_{j=1}^{n} d x^{j} \wedge d \theta^{j} .
$$


The moment polytope $P_{X}$ is a Delzant polytope (see [D88] or p. 698 of [DP09]) determined by a set of inequalities $\left\{\ell_{j}(x) \geq 0\right\}_{j=1, \ldots, r}$, where $r$ is the number of facets of $P_{X}$ and for each $j=1, \ldots, r$,

$$
\ell_{j}(x)=\nu_{j} \cdot x+\lambda_{j}
$$

where $\nu_{j}$ is the (inward pointing) primitive integral vector normal to the $j$ th facet of $P_{X}$, and $\lambda_{j} \in \mathbb{R}$.

We now describe the coordinate chart associated to a vertex $v \in P_{X}$. Since we assume $X$ is smooth, the polytope is regular; that is, there are $n$ facets adjacent to each vertex, with normal vectors forming a $\mathbb{Z}$-basis of $\mathbb{Z}^{n}$. Reorder (if necessary) the inequalities so that the first $n$ correspond to the facets adjacent to $v$. Then $\ell_{1}(v)=\ell_{2}(v)=\cdots=\ell_{n}(v)=0$. Let $A_{v} \in G L_{n}(\mathbb{Z})$ be the matrix whose rows are the vectors $\nu_{j}$, and let $\lambda_{v}={ }^{t}\left(\lambda_{1}, \ldots, \lambda_{n}\right)$. Define new (vertex action-angle) coordinates $x_{v}$ on $\mathbb{R}^{n}$ and $\theta_{v}$ on $\mathbb{T}^{n}$ by

$$
x_{v}:=A_{v} x+\lambda_{v}, \quad \text { and } \quad \theta_{v}:={ }^{t} A_{v}^{-1} \theta .
$$

The image of the polytope $P_{X}$ under $x_{v}$ in (2.3) is also a Delzant polytope $P_{X}^{v}$

$$
P_{X}^{v}=A_{v} P_{X}+\lambda_{v}
$$

with the vertex $v$ mapped to the origin and the codimension one faces meeting at the origin contained in the coordinate hyperplanes. Given a different vertex $v^{\prime}$, with associated matrix $A_{v^{\prime}} \in G L_{n}(\mathbb{Z})$, and vector $\lambda_{v^{\prime}}$, the transition functions between the corresponding vertex action angle coordinates read

$$
\begin{aligned}
& x_{v^{\prime}}=A_{v^{\prime}} A_{v}^{-1}\left(x_{v}-\lambda_{v}\right)+\lambda_{v^{\prime}}, \\
& \theta_{v^{\prime}}={ }^{t} A_{v^{\prime}}^{-1}{ }^{t} A_{v} \theta_{v} .
\end{aligned}
$$

For a face $F \subset P_{X}$ (that is, a linear boundary component of any codimension, including the polytope itself, the facets and the edges), denote by $\check{F}$ the interior of $F$, with the convention that $\{\tilde{v}\}=\{v\}$ for vertices. The vertex chart neighborhood at $v$ is defined to be the following $\mathbb{T}^{n}$-invariant open set

$$
U_{v}:=\mu^{-1}\left(\bigcup_{\text {faces } F \text { of } P_{X} \text { adjacent to } v} \check{F}\right) .
$$

We consider on $U_{v}$ coordinates $\left\{a_{v}^{j}, b_{v}^{j}\right\}_{j=1, \ldots, n}$ related to the vertex actionangle coordinates $\left\{x_{v}^{j}, \theta_{v}^{j}\right\}_{j=1, \ldots, n}$ by $a_{v}^{j}+\mathrm{i} b_{v}^{j}=\sqrt{x_{v}^{j}} \mathrm{e}^{\mathrm{i} \theta_{v}^{j}}, j=1, \ldots, n$, on $\check{X}$ (see, for example, Sections 3 and 4 of [DP09]). Since $x_{v}$ takes values in the polytope $P_{X}^{v} \subset \mathbb{R}^{n}(2.4)$, (it is surjective to the polytope minus the faces not containing the origin) and $\theta_{v} \in \mathbb{R}^{n} / \mathbb{Z}^{n}$, the image of $U_{v}$ under $a_{v}+\mathrm{i} b_{v}$ is a bounded neighborhood of the origin in $\mathbb{C}^{n}$. The (non-holomorphic) transition 
functions between coordinate functions $a_{v}+\mathrm{i} b_{v}$ and $a_{v^{\prime}}+\mathrm{i} b_{v^{\prime}}$ for vertices $v$ and $v^{\prime}$ can be obtained from (2.5) (see Section 4 of [DP09]),

$$
\begin{aligned}
a_{v^{\prime}}^{j^{\prime}}+\mathrm{i} b_{v^{\prime}}^{j^{\prime}}= & \sqrt{\sum_{j=1}^{n}\left(A_{v^{\prime}} A_{v}^{-1}\right)_{j^{\prime} j}\left(\left|a_{v}^{j}+\mathrm{i} b_{v}^{j}\right|^{2}-\lambda_{v}^{j}\right)+\lambda_{v^{\prime}}^{j^{\prime}}} \\
& \times \prod_{j=1}^{n}\left(\frac{a_{v}^{j}+\mathrm{i} b_{v}^{j}}{\left|a_{v}^{j}+\mathrm{i} b_{v}^{j}\right|}\right)^{\left({ }^{t} A_{v^{\prime}}^{-1}{ }^{t} A_{v}\right)_{j^{\prime} j}} \cdot
\end{aligned}
$$

We will also need the much simpler transition functions for holomorphic vertex coordinates (which will be introduced below in the section on Kähler structures).

We note that the faces of $P_{X}$ correspond to points in $X$ with non-trivial stabilizer as follows: suppose $F$ is a face adjacent to $v$ given by $\left\{x_{v}^{j_{s}}=\right.$ $0\}_{s=1, \ldots, j_{F}}$ (so $F$ is a codimension- $j_{F}$ face). Then the points in $\mu^{-1}(F)$ are fixed by the subtorus parameterized by the coordinates $\left\{\left(\theta_{v}^{j_{1}}, \ldots, \theta_{v}^{j_{F}}\right)\right\}$. Let $V$ be the set of vertices of $P_{X}$. We call $\left\{\left(\mu^{-1}\left(\check{P}_{X}\right),(x, \theta)\right),\left(U_{v},\left(a_{v}, b_{v}\right)\right): v \in\right.$ $V$ \} the vertex atlas of $X$.

The symplectic form in the vertex coordinate chart $U_{v}$ can be computed by pullback of (2.2) under the coordinate change (2.3) to be

$$
\left.\omega\right|_{U_{v}}=\sum_{j=1}^{n} 2 d a_{v}^{j} \wedge d b_{v}^{j}
$$

and on $U_{v} \cap \check{X}=\check{X}$

$$
\left.\omega\right|_{\check{X}}=\sum_{j=1}^{n} d x_{v}^{j} \wedge d \theta_{v}^{j} .
$$

2.2.2. The Kähler structure of $\boldsymbol{X}$. In order to describe the toric Kähler structures on $X$, let us consider torus-invariant complex structures on the symplectic toric manifold $(X, \omega)$ with moment polytope $P_{X}$. Let $g_{P_{X}} \in$ $C^{\infty}\left(\check{P}_{X}\right)$ be

$$
g_{P_{X}}(x)=\frac{1}{2} \sum_{j=1}^{r} \ell_{j}(x) \log \ell_{j}(x) .
$$

Definition 2.7. Let $C_{P_{X}}^{\infty}\left(P_{X}\right)$ be the set of smooth functions on $P_{X}$ such that $\varphi \in C_{P_{X}}^{\infty}\left(P_{X}\right)$ if $\operatorname{Hess}_{x}\left(g_{P_{X}}+\varphi\right)$ is positive definite on $\check{P}_{X}$ and there exists a strictly positive function $\alpha \in C^{\infty}\left(P_{X}\right)$ so that

$$
\operatorname{det}\left(\operatorname{Hess}_{x}\left(g_{P_{X}}+\varphi\right)\right)=\left(\alpha(x) \prod_{j=1}^{r} \ell_{j}(x)\right)^{-1}
$$

on $\check{P}_{X}$. 
A torus-invariant complex structure on $(X, \omega)$ is determined by a symplectic potential

$$
g=g_{P_{X}}+\varphi,
$$

with $\varphi \in C_{P_{X}}^{\infty}\left(P_{X}\right)$, see Section 4 of [Gui94] and Theorem 2.8 of [Abr03]. In the symplectic frame determined by the action-angle coordinates $(x, \theta)$ on $\check{X}$, the toric complex structure $I$ and the metric $\gamma=\omega(\cdot, I \cdot)$ tensors associated to the symplectic potential $g$ are then

$$
I=\left(\begin{array}{cc}
0 & -G^{-1} \\
G & 0
\end{array}\right) \quad \text { and } \quad \gamma=\left(\begin{array}{cc}
G & 0 \\
0 & G^{-1}
\end{array}\right)
$$

where $G=\operatorname{Hess}_{x} g$ is the Hessian of $g$.

Let us now relate these complex structures to the algebro-geometric description of toric manifolds. By a standard construction, see, for example, Section 5 in $[\mathbf{D 8 8}]$ or Definition 6.4.2 in $[\mathbf{C d S}]$, associated to the moment polytope $P_{X}$ there is an associated complete fan $\Sigma$. This fan defines a compact smooth toric variety $Y$ diffeomorphic to $X$ (see below) and with canonical complex structure defined by $\Sigma$.

The complex torus $\left(\mathbb{C}^{*}\right)^{n}$ acts on $Y$ with a dense open orbit (biholomorphic to $\left.\left(\mathbb{C}^{*}\right)^{n}\right)$ which we henceforth refer to as the open orbit. Let $M$ denote the (integer lattice of) characters of $\left(\mathbb{C}^{*}\right)^{n}$, so that after a choice of basis, $M \simeq \mathbb{Z}^{n} .{ }^{1}$ The characters of $\left(\mathbb{C}^{*}\right)^{n}$ extend to meromorphic functions on $Y$ with torus-invariant divisors.

The toric variety $Y$ has an atlas of holomorphic coordinates $\left\{\left(V_{v}, \tilde{w}_{v}\right)\right\}_{v \in V}$, $\tilde{w}_{v}=\left(\tilde{w}_{v}^{1}, \ldots, \tilde{w}_{v}^{n}\right)$, where for each pair of vertices $v, v^{\prime}$, over $V_{v} \cap V_{v^{\prime}}$ the glueing conditions are given by

$$
\tilde{w}_{v^{\prime}}=\tilde{w}_{v}^{A_{v} A_{v^{\prime}}^{-1}}
$$

with $A_{v} A_{v^{\prime}}^{-1}$ interpreted as a row of multiindices; i.e., $\tilde{w}_{v^{\prime}}^{j}=\prod_{l=1}^{n}\left(\tilde{w}_{v}^{l}\right)^{\left(A_{v} A_{v^{\prime}}^{-1}\right)_{j}^{l}}$. (See, for example, Section 5 of [DP09].)

Denote the open orbit in $Y$ by $V_{0}$. The symplectic potential $g$ fixes a biholomorphism $(\check{X}, I) \cong \check{P_{X}} \times \mathbb{T}^{n} \cong V_{0} \cong\left(\mathbb{C}^{*}\right)^{n}$ given by

$$
\begin{aligned}
\check{P}_{X} \times \mathbb{T}^{n} & \longrightarrow V_{0} \simeq\left(\mathbb{C}^{*}\right)^{n} \\
(x, \theta) & \mapsto \tilde{w}=\mathrm{e}^{y+\mathrm{i} \theta}=\left(\mathrm{e}^{y^{1}+\mathrm{i} \theta^{1}}, \ldots, \mathrm{e}^{y^{n}+\mathrm{i} \theta^{n}}\right),
\end{aligned}
$$

where $y^{j}=\partial g / \partial x^{j}$. Note that this map is not a symplectomorphism with respect to the standard symplectic structure on $\left(\mathbb{C}^{*}\right)^{n}$.

The map $x \mapsto y=\partial g / \partial x$ is a bijective Legendre transform. The inverse map is given by $x=\partial h / \partial y$, where $h$ is a Kähler potential given in terms of $g$ by

$$
h:=x \cdot y-g .
$$

\footnotetext{
${ }^{1}$ We will henceforth identify $M \cong \mathbb{Z}^{n}$ and $M \otimes \mathbb{R} \cong \mathbb{R}^{n}$.
} 
This biholomorphism extends uniquely to a biholomorphism

$$
\psi_{g}: X \rightarrow Y
$$

as follows. The complex structure associated to $g$ defines holomorphic coordinates in the vertex coordinate charts via the coordinate change (2.3) to yield

$$
\begin{aligned}
U_{v} & \longrightarrow V_{v} \\
\left(x_{v}, \theta_{v}\right) & \mapsto \tilde{w}_{v}=\mathrm{e}^{y_{v}+\mathrm{i} \theta_{v}},
\end{aligned}
$$

where $y_{v}^{j}:=\partial g / \partial x_{v}^{j}=\sum_{k=1}^{n}\left(A_{v}^{-1}\right)_{j}^{k} \partial g / \partial x^{k}$. Using the observation that $y_{v}+\mathrm{i} \theta_{v}={ }^{t} A_{v}^{-1}(y+\mathrm{i} \theta)$, one may verify that (2.9) is indeed satisfied. Similarly, on $V_{0}$ we have

$$
\tilde{w}=\tilde{w}_{v}^{A_{v}}
$$

which will be useful below.

We define the $I$-dependent holomorphic vertex atlas $\left\{\left(U_{v}, w_{v}\right)\right\}_{v \in V}$ on $X$ to be the pullback by $\psi_{g}$ of the holomorphic atlas $\left\{\left(V_{v}, \tilde{w}_{v}\right)\right\}_{v \in V}$ on $Y$. (We will also denote the pullback of the chart $\left(V_{0}, \tilde{w}\right)$ on $Y$ to $X$ by $\left(U_{0}, w\right)$.) The $I$-dependent transition functions for the holomorphic coordinate charts $\left(U_{v}, w_{v}\right), v \in V,\left(U_{0}, w\right)$ on $X$ are therefore the pullbacks by $\psi_{g}$ of the corresponding transition functions on $Y$ in (2.9) and (2.12).

Henceforth, we will assume that $X$ is equipped with a Kähler structure determined by $\omega$ and by a symplectic potential $g=g_{P_{X}}+\varphi, \varphi \in C_{P_{X}}^{\infty}\left(P_{X}\right)$.

2.2.3. Line bundles and sections. Since compact smooth toric varieties are simply connected, the Picard group of equivalence classes of holomorphic line bundles is isomorphic to $H^{2}(X, \mathbb{Z})$, with isomorphism established by the first Chern class. In other words, fixing the first Chern class of a line bundle on the the complex toric manifold $X$ fixes the bundle up to isomorphism. (See the Corollary on p. 64, on Section 3.4 of $[\mathbf{F}]$.)

The linear equivalence classes of the torus-invariant divisors of $X$ generate the Picard group of $X$, and there is a one-to-one correspondence between irreducible torus-invariant divisors and 1-cones in $\Sigma$ (see, Part I, Chapter 4 of $\left[\right.$ CLS11]). Denote the set of 1-cones in $\Sigma$ by $\Sigma^{(1)}$. The $j$ th 1-cone in $\Sigma^{(1)}$ is generated by the primitive integral vector $\nu_{j}$ normal to the $j$ th facet of $P_{X}$. Then, the associated irreducible divisor $D_{j}=\mu^{-1}\left(\left\{x \in P_{X}: \ell_{j}(x)=\right.\right.$ $\left.\left.\nu_{j} \cdot x+\lambda_{j}=0\right\}\right)$ is the inverse image under the moment map $\mu$ of that facet of $P_{X}$. The Picard group is then generated by the linear equivalence classes of irreducible divisors $D_{1}, \ldots, D_{r}$. Consider a divisor $D^{L}=\lambda_{1}^{L} D_{1}+\cdots+\lambda_{r}^{L} D_{r}$, for $\lambda_{1}^{L}, \ldots, \lambda_{r}^{L} \in \mathbb{Z}$, defining a holomorphic line bundle $L=\mathcal{O}\left(D^{L}\right)$ and a (unique up to constant) meromorphic section of $L$ with divisor $D^{L}, \sigma_{D^{L}}$. 
From [CLS11], the divisor of the (rational) function defined on the open orbit by $w^{m}, m \in \mathbb{Z}^{n}$, can be computed to be

$$
\operatorname{div}\left(w^{m}\right)=\sum_{j=1}^{r}\left\langle\nu_{j}, m\right\rangle D_{j} .
$$

Then, we have

$$
\begin{aligned}
H^{0}(X, L) & =\operatorname{span}_{\mathbb{C}}\left\{w^{m} \sigma_{D^{L}}: m \in \mathbb{Z}^{n}, \operatorname{div}\left(w_{0}^{m} \sigma_{D^{L}}\right) \geq 0\right\} \\
& =\operatorname{span}_{\mathbb{C}}\left\{w^{m} \sigma_{D^{L}}: m \in \mathbb{Z}^{n},\left\langle m, \nu_{i}\right\rangle+\lambda_{i}^{L} \geq 0, i=1, \ldots, r\right\} .
\end{aligned}
$$

Therefore, there is a natural bijection between a basis of $H^{0}(X, L)$ whose elements are weight vectors for the action of the torus and the integral points of the Delzant polytope with integral vertices ${ }^{2}$

$$
P_{L}:=\left\{x \in \mathbb{R}^{n}:\left\langle x, \nu_{j}\right\rangle+\lambda_{j}^{L} \geq 0, j=1, \ldots, r\right\} \subset \mathbb{R}^{n} .
$$

For simplicity, let us assume that $L$ is ample so that there is a canonical bijection between the vertices of $P_{L}$ and the vertices of $P_{X}$, defined by the equality of the set of normals of the facets meeting at those vertices. (In fact, if $L$ is ample there is a bijection between the faces of $P_{X}$ and of $P_{L}$. See Section 3.2.1 of $[\mathbf{C K}]$.) Let us denote by the same symbol $v$ a vertex of $P_{L}$ and the corresponding vertex of $P_{X}$. The holomorphic section corresponding to the vertex $v$ of $P_{L}$ will provide a local trivializing section on the open set $U_{v}$, so that one obtains a global system of local holomorphic trivializations for $L$. For such vertex $v$, we can order the inequalities $\left\{\ell_{j}^{L}(m):=\left\langle m, \nu_{j}\right\rangle+\lambda_{j}^{L} \geq 0, j=1 \ldots r\right\}$ so that $\nu_{1}, \ldots, \nu_{n}$ are the normals to the facets of $P_{L}$ meeting at $v$; this is the same ordering that we used in the definition of the vertex coordinates on $X$. Using this ordering, we set $\lambda_{v}^{L}={ }^{t}\left(\lambda_{v, 1}^{L}, \ldots, \lambda_{v, n}^{L}\right):=\left(\lambda_{1}^{L}, \ldots, \lambda_{n}^{L}\right)$.

The holomorphic section corresponding to a vertex $v$ of $P_{L}$ is given by

$$
\mathbf{1}_{v}:=w_{v}^{-\lambda_{v}^{L}} \sigma_{D^{L}} .
$$

Using (2.12) and (2.13), one obtains that the divisor of the meromorphic function $w_{v}^{\lambda_{v}^{L}}$ on $U_{v}$ is

$$
\operatorname{div}_{U_{v}}\left(w_{v}^{\lambda_{v}^{L}}\right)=\left(\lambda_{v, 1}^{L} D_{1}+\cdots+\lambda_{v, n}^{L} D_{n}\right) \cap U_{v}
$$

and therefore $\operatorname{div}_{U_{v}}\left(\mathbf{1}_{v}\right)=0$, so that $\mathbf{1}_{v}$ is a trivializing holomorphic section of $L$ on $U_{v}=X \backslash\left\{\cup_{j=n+1}^{r} D_{j}\right\}$. We remark that these sections are determined up to a constant by their divisors and they are therefore defined for every line bundle in the isomorphism class of $L$.

\footnotetext{
${ }^{2}$ Note that we have different sign conventions for $\lambda_{F}$ than those used in [BFMN11]. We have chosen rather to follow the convention in [CLS11], as they seem to make certain equations more natural (for example, shifting $\lambda_{F} \mapsto \lambda_{F}+1$ has the effect of shifting the facet $F$ one unit along the outward pointing normal to $F$ ).
} 
For notational convenience, let $\mathbf{1}_{0}=\sigma_{D^{L}}$. Using (2.16), we may compute the transition functions for $L$ relative to the holomorphic vertex atlas obtaining, $g_{v^{\prime} v}^{L}:=\mathbf{1}_{v} / \mathbf{1}_{v^{\prime}}=w_{v^{\prime}}^{\lambda_{v^{\prime}}^{L}} / w_{v}^{\lambda_{v}^{L}}$ and $g_{0 v}^{L}:=\mathbf{1}_{v} / \mathbf{1}_{0}=w_{v}^{-\lambda_{v}^{L}}$. Combined with (2.9), the transition functions for $\mathcal{O}\left(\lambda_{1}^{L} D_{1}+\cdots+\lambda_{r}^{L} D_{r}\right)$ become

$$
\begin{gathered}
g_{v 0}^{L}(w)=w^{A_{v}^{-1} \lambda_{v}^{L}} \text { and } \\
g_{v^{\prime} v}^{L}\left(w_{v^{\prime}}\right)=w_{v^{\prime}}^{\lambda_{v^{\prime}}^{L}-A_{v^{\prime}} A_{v}^{-1} \lambda_{v}^{L}} .
\end{gathered}
$$

Remark 2.8. Note that the transition functions of $L$ depend on the variation of complex structure on $X$ through the symplectic potential $g$, since $w=\mathrm{e}^{\frac{\partial g}{\partial x}+\mathrm{i} \theta}$ and $w_{v}=\mathrm{e}^{\frac{\partial g}{\partial x_{v}}+\mathrm{i} \theta_{v}}$ (see (2.10) and (2.11)). We will consider one-parameter families of symplectic potentials, $g_{s}=g_{P_{X}}+\varphi+$ $s \psi, \varphi, \psi \in C_{P_{X}}^{\infty}\left(P_{X}\right), s \in \mathbb{R}^{+}$. The transition functions and therefore $L$ depend smoothly on $s$.

These relations define a holomorphic line bundle on $X$ for any integral values of $\lambda_{i}^{L}$, even if this line bundle is not ample. In this case, sections of the sheaf of holomorphic sections over $U_{v}$ are defined as in (2.14) with $X$ replaced by $U_{v}$ and the divisors $D$ replaced by $D \cap U_{v}$.

Using the transition functions (2.17) for $L$, we can give it a concrete realization as the following equivariant line bundle:

$$
L=\left(\bigsqcup_{v \in V} U_{v} \times \mathbb{C}\right) / \sim,
$$

where $(w, z) \sim\left(w^{\prime}, z^{\prime}\right)$ if $w=w^{\prime} \in U_{v} \cap U_{v^{\prime}}$ and $z=g_{v v^{\prime}}^{L}(w) z^{\prime}$. We will assume that $L=\mathcal{O}(D), D=\sum_{j=1}^{r} \lambda_{j}^{L} D_{j}$, is the line bundle defined by (2.18). In each open set in the holomorphic vertex atlas, the trivializing sections $\mathbf{1}_{v}$ (or $\mathbf{1}_{0}$ on the open orbit) defined above are given by

$$
\mathbf{1}_{v}(w):=[(w, 1)], \quad w \in U_{v}\left(\text { or } w \in U_{0}, \text { for the open orbit }\right) .
$$

For $\sigma \in \Gamma(L)$ denote by $\sigma_{v}, \sigma_{0}$ its components on the local frames given, respectively, by $\mathbf{1}_{v}, \mathbf{1}_{0}$. For an integral point $m \in P_{L} \cap \mathbb{Z}^{n}$, we denote by $\sigma^{m}$ the holomorphic section with $\sigma_{0}^{m}=w^{m}$. Using the transition functions, we obtain expressions for $\sigma^{m}$ in the holomorphic vertex charts:

$$
\sigma_{v}^{m}\left(w_{v}\right)=w_{v}^{\ell_{v}(m)} \mathbf{1}_{v}
$$

where $\ell_{v}(x):=A_{v} x+\lambda_{v}^{L}$.

We have

Lemma 2.9. Let $L$ be the equivariant holomorphic line bundle defined by (2.17) and (2.18). The unitarization $L^{U(1)}$ associated with $L$ defined over a compact toric variety $X$ has complex structure independent transition functions on the vertex atlas. 
Proof. Recall from Section 2.1 that the unitarization of $L$ is the line bundle $L^{U(1)}$ with local trivializing sections $\mathbf{1}_{v}^{U(1)}$ on the vertex charts and $\mathbf{1}_{0}^{U(1)}$ on the open orbit. The corresponding transition functions are

$$
\begin{aligned}
\tilde{g}_{v^{\prime} v}^{L} & :=\mathbf{1}_{v}^{U(1)} / \mathbf{1}_{v^{\prime}}^{U(1)}=\mathrm{e}^{\mathrm{i}\left(\lambda_{v^{\prime}}^{L}-A_{v^{\prime}} A_{v}^{-1} \lambda_{v}^{L}\right) \cdot \theta_{v^{\prime}}} \text { and } \\
\tilde{g}_{0 v}^{L} & :=\mathbf{1}_{v}^{U(1)} / \mathbf{1}_{0}^{U(1)}=\mathrm{e}^{-\mathrm{i}\left(A_{v}^{-1} \lambda_{v}^{L}\right) \cdot \theta} .
\end{aligned}
$$

We see that, unlike those for $L$ itself, these transition functions are complex structure independent.

Recall also from Remark 2.1 that a Hermitian structure on $L$ defines an isomorphism between $L$ and $L^{U(1)}$.

2.2.4. The canonical bundle. The complex structure $I$ on $(X, \omega)$ (see (2.8)) defines the canonical holomorphic line bundle $K_{I}:=\bigwedge^{n}\left(T^{*}\right)^{1,0}$, whose sections are $(n, 0)$-forms. Consider, in the open orbit $U_{0}$, the $I$-holomorphic $(n, 0)$-form $d Z=d z^{1} \wedge \cdots \wedge d z^{n}=d W / w^{\mathbf{1}}$, where

$$
z={ }^{t}\left(z^{1}, \ldots, z^{n}\right)=\partial g / \partial x+\mathrm{i} \theta
$$

and $d W:=d w^{1} \wedge \cdots \wedge d w^{n}$, so that $d Z$ and $d W$ are trivializing sections of $K_{\left.I\right|_{U_{0}}} \cdot\left(\right.$ Here, $\mathbf{1}=(1, \ldots, 1)$ so that $w^{\mathbf{1}}=w^{1} \cdots w^{n}$.) Then

Lemma 2.10. [CLS11, Sec. 8.2] The (n,0)-form given on the open orbit by $d Z$ extends to a meromorphic section of $K_{I}$ with divisor $\operatorname{div}(d Z)=-D_{1}-$ $\cdots-D_{r}$. On the holomorphic vertex chart $U_{v}$ this section is proportional to $d W_{v} / w_{v}^{\mathbf{1}}=d Z_{v}$.

Since the $w_{v}^{j}$ 's are holomorphic coordinates on the chart $U_{v}$, it follows that a system of local holomorphic trivializations for $K_{I}$ is given by $\left\{\left(U_{v}, d W_{v}\right)\right\}$. Relative to this system of trivializations of $K_{I}$, the transition functions are computed to be

$$
g_{v^{\prime} v}^{K_{I}}=w_{v^{\prime}}^{-\mathbf{1}+A_{v^{\prime}} A_{v}^{-1} \mathbf{1}},
$$

so that, as expected from the form of $\operatorname{div}(d Z), K_{I}$ is isomorphic to a line bundle of the form of (2.18).

From Section 2.1, we have that the equivariant Hermitian holomorphic line bundle $K_{I}$ admits a decomposition $K_{I}^{U(1)} \otimes\left|K_{I}\right|$. The unitarization $K^{U(1)}$ is trivialized by

$$
\left\{\left(U_{v}, \frac{d W_{v}}{\left|d W_{v}\right|}\right)\right\}_{v \in V}
$$

with corresponding transition functions

$$
\tilde{g}_{v^{\prime} v}^{K_{I}}=\mathrm{e}^{\mathrm{i}\left(-\mathbf{1}+A_{v} A_{v^{\prime}}^{-1} \mathbf{1}\right) \cdot \theta_{v^{\prime}}} .
$$


We see that, in accordance with Lemma $2.9, K_{I}^{U(1)}$ has $I$-independent transition functions on the vertex atlas. On the other hand, note that the line bundles $K_{I}^{U(1)}$ depend on $I$ because their fibers change with $I$ (see (2.23)). In order to facilitate the study of the dependence of polarized sections on the complex structure $I$, it will be convenient to consider a line bundle with the same transition functions as $K_{I}^{U(1)}$ but defined as in (2.18), so that this line bundle is $I$-independent (but has an $I$-dependent isomorphism to $\left.K_{I}^{U(1)}\right)$.

Definition 2.11. Denote by $\widetilde{K}^{U(1)}$ the (I-independent) equivariant line bundle defined as in (2.18) with $U(1)$-valued transition functions given by $(2.24)$.

In the remainder of this section, we continue to consider a fixed toric complex structure $I$, obtained from a symplectic potential $g$, and will drop the subscript $I$ for simplicity. $K$ has a canonical Hermitian structure given by comparison with the Liouville volume form, that is, for an $(n, 0)$-form $\eta$,

$$
\|\eta\|_{K}^{2}:=\frac{\eta \wedge \bar{\eta}}{(2 \mathrm{i})^{n}(-1)^{n(n+1) / 2} \omega^{n} / n !} .
$$

Let $\nabla^{K}$ denote the Chern connection corresponding to this Hermitian structure. In the above trivialization, we can compute the connection 1-form of the Chern connection (using Lemma 2.6) to be

$$
\partial \log \|d Z\|_{K}^{2} .
$$

Lemma 2.12. $\|d Z\|_{K}^{2}=\operatorname{det} G$, where $G=\operatorname{Hess}_{x} g$. Hence, the Chern connection 1-form in the open orbit $U_{0}$ is $\Theta_{0}=\partial \log \operatorname{det} G$.

Proof. Since $z^{j}=\partial g / \partial x^{j}+\mathrm{i} \theta^{j}$, we see that $d z=G d x+\mathrm{i} d \theta$ and similarly that $d \bar{z}=G d x-\mathrm{i} d \theta$. We can express these in the matrix equation

$$
\left(\begin{array}{l}
d z \\
d \bar{z}
\end{array}\right)=\left(\begin{array}{cc}
G & \mathrm{i} 1 \\
G & -\mathrm{i} 1
\end{array}\right)\left(\begin{array}{l}
d x \\
d \theta
\end{array}\right)
$$

when

$$
\begin{aligned}
\|d Z\|_{K}^{2} & =\frac{d Z \wedge d \bar{Z}}{(-2 \mathrm{i})^{n}\left(d x^{1} \wedge \cdots \wedge d x^{n} \wedge d \theta^{1} \wedge \cdots \wedge d \theta^{n}\right)} \\
& =\frac{1}{(-2 \mathrm{i})^{n}} \operatorname{det}\left(\begin{array}{cc}
G & \mathrm{i} \mathbf{1} \\
G & -\mathrm{i} \mathbf{1}
\end{array}\right)=\operatorname{det} G .
\end{aligned}
$$

Similarly, we have

$$
\left\|d Z_{v}\right\|_{K}^{2}=\operatorname{det} G_{v},
$$

where $G_{v}=\operatorname{Hess}_{x_{v}} g$. 
The curvature of the Chern connection is easily computed, giving

$$
F_{\nabla^{K}}=\bar{\partial} \partial \log \operatorname{det} G .
$$

Let $\operatorname{Ric}_{\gamma}$ denote the Ricci curvature tensor of the metric $\gamma=\omega(\cdot, I \cdot)$, and let $\rho=\operatorname{Ric}(I \cdot, \cdot)$ be the corresponding Ricci form. Then by [Mor07, Prop. 11.4] we have

$$
F_{\nabla^{K}}=\mathrm{i} \rho \text {. }
$$

Note that this implies $c_{1}(K)=-c_{1}(X)=-[\rho / 2 \pi]$.

Lemma 2.13. The section $d W_{v}=w_{v}^{1} d Z_{v}$ is a nowhere vanishing holomorphic section of $K$ on the holomorphic vertex chart $U_{v}$ and, in the induced trivializations, the Chern connection 1 -forms on $K^{U(1)}$ and $|K|$ are

$$
\begin{aligned}
\Theta_{v}^{K^{U(1)}} & =i \sum_{k=1}^{n} d \theta_{v}^{k}+\frac{\mathrm{i}}{2}\left(\frac{\partial}{\partial x_{v}} \log \operatorname{det} G_{v}\right) \cdot G_{v}^{-1} d \theta_{v}, \quad \text { and } \\
\Theta_{v}^{|K|} & =\frac{1}{2}\left(\frac{\partial}{\partial x_{v}} \log \operatorname{det} G_{v}\right) \cdot d x_{v}+G_{v} d x_{v} .
\end{aligned}
$$

Remark 2.14. The following expressions for the connection 1-forms for the induced connections $\nabla^{K^{U(1)}}$ and $\nabla^{|K|}$ over the open orbit will also be useful below:

$$
\begin{aligned}
\Theta_{0}^{K^{U(1)}} & =\operatorname{iIm} \Theta=\frac{\mathrm{i}}{2}\left(\frac{\partial}{\partial x} \log \operatorname{det} G\right) \cdot G^{-1} d \theta, \text { and } \\
\Theta_{0}^{|K|} & =\operatorname{Re} \Theta=\frac{1}{2}\left(\frac{\partial}{\partial x} \log \operatorname{det} G\right) \cdot d x .
\end{aligned}
$$

Proof. If $f$ is real valued, then

$$
\operatorname{Im} \partial f=\frac{1}{2}\left(\frac{\partial f}{\partial x} \cdot G^{-1} d \theta-\frac{\partial f}{\partial \theta} \cdot G d x\right)
$$

and

$$
\operatorname{Re} \partial f=\frac{1}{2}\left(\frac{\partial f}{\partial x} \cdot d x+\frac{\partial f}{\partial \theta} \cdot d \theta\right)
$$

with similar formulas in the vertex charts (with $x^{j}$ and $\theta^{j}$ replaced by $x_{v}^{j}$ and $\theta_{v}^{j}$ ). With $f=\log \operatorname{det} G$ (observing that $f=f(x)$ ) we obtain, from $\Theta_{0}=\partial \log \operatorname{det} G$, the open orbit 1-forms (2.28).

Next, by [CLS11], the "canonical" section $d Z=d W / w^{1}$ (which has the same representation $d W_{v} / w_{v}^{1}$ in the holomorphic vertex charts) has simple poles along each torus-invariant divisor. To obtain a trivializing section on the holomorphic vertex chart $U_{v}$, we multiply by a factor with simple zeroes along the divisors adjacent to $v$ and thus arrive at the desired combination $w_{v}^{1} d Z_{v}$. 
To obtain the connection 1 -forms in the chart $U_{v}$ with respect to $w_{v}^{\mathbf{1}} d Z_{v}$, we first recall the norm

$$
\left\|w_{v}^{\mathbf{1}} d Z_{v}\right\|^{2}=\left|w_{v}^{\mathbf{1}}\right|^{2} \operatorname{det} G_{v} .
$$

From this, noting that $\operatorname{det} G_{v}$ is a constant multiple of $\operatorname{det} G$, we see that the Chern connection 1-form on $U_{v}$ is

$$
\begin{aligned}
\Theta_{v}=\partial \log \left(\left|w_{v}^{\mathbf{1}}\right|^{2} \operatorname{det} G_{v}\right) & =\partial \log \operatorname{det} G_{v}+\sum_{j} \partial \log w_{v}^{j} \\
& =\partial \log \operatorname{det} G_{v}+\sum_{j} d z_{v}^{j} .
\end{aligned}
$$

Since $d z_{v}=G_{v} d x_{v}+\mathrm{i} d \theta_{v}$, using (2.29) and that $G$ depends only on $x$, we obtain the connection 1-form

$$
\begin{aligned}
\Theta_{v}^{K^{U(1)}} & =\mathrm{i} \operatorname{Im}\left(\partial \log \operatorname{det} G_{v}+\sum_{j} d z_{v}^{j}\right) \\
& =\frac{\mathrm{i}}{2}\left(\frac{\partial}{\partial x} \log \operatorname{det} G_{v}\right) \cdot G_{v}^{-1} d \theta_{v}+\mathrm{i} \sum_{j=1}^{n} d \theta_{v}^{j}
\end{aligned}
$$

as desired.

Similarly, using (2.30) we obtain the connection 1-form

$$
\Theta_{v}^{|K|}=\operatorname{Re}\left(\partial \log \operatorname{det} G_{v}+\sum_{j} d z_{v}^{j}\right)=\frac{1}{2}\left(\frac{\partial}{\partial x_{v}} \log \operatorname{det} G_{v}\right) \cdot d x_{v}+G_{v} d x_{v}
$$

as desired.

Note that although $K_{I}^{U(1)}$ has complex structure independent transition functions, its Chern connection depends on $I$.

\section{Half-form corrected Kähler quantization}

3.1. Motivation. Suppose the square root $\sqrt{K_{I}}$ of the corresponding canonical bundle exists so that, in particular, $c_{1}(X) / 2$ is integral. Assume, moreover, that $[\omega / 2 \pi] \in H^{2}(X, \mathbb{Z})$ and let $\ell \rightarrow X$ be a (smooth) Hermitian line bundle with compatible connection with curvature given by $-\mathrm{i} \omega$, that is, $\ell$ is a prequantum line bundle. More specifically, let $\ell$ be an equivariant line bundle defined as in (2.18), with $U(1)$-valued transition functions on the vertex atlas $(2.20)$

$$
\tilde{g}_{v v^{\prime}}^{\ell}=\mathrm{e}^{\mathrm{i}\left(A_{v^{\prime}} A_{v}^{-1} \lambda_{v}-\lambda_{v^{\prime}}\right) \cdot \theta_{v^{\prime}}}, \quad \text { and } \quad \tilde{g}_{v 0}^{\ell}=\mathrm{e}^{\mathrm{i} \lambda_{v} \cdot \theta_{v}},
$$


with $\lambda_{v} \in \mathbb{Z}$ suitably chosen to have $c_{1}(\ell)=\left[\frac{\omega}{2 \pi}\right]$. Equip $\ell$ with the $U(1)$ connection $\nabla^{\ell}$ given by the connection forms

$$
\begin{aligned}
& \Theta_{0}^{\ell}=\frac{\nabla^{\ell} \mathbf{1}_{0}^{U(1)}}{\mathbf{1}_{0}^{U(1)}}=\mathrm{i} x \cdot d \theta \text { on } \check{X}, \\
& \Theta_{v}^{\ell}=\frac{\nabla^{\ell} \mathbf{1}_{v}^{U(1)}}{\mathbf{1}_{v}^{U(1)}}=\mathrm{i} x_{v} \cdot d \theta_{v} \text { on } U_{v}, \quad v \in V .
\end{aligned}
$$

One may easily check that $\left\{\Theta_{0}^{\ell}, \Theta_{v}^{\ell}: v \in V\right\}$ does indeed define a $U(1)$ connection on $\ell$; see the comment following (3.10).

Since the square of a (local) section $\eta \in \Gamma\left(\sqrt{K_{I}}\right)$ can be identified with a (local) section of $K_{I}$, the line bundle $\sqrt{K_{I}}$ inherits a Hermitian structure from that of $K_{I}$ given by [Woo91]

$$
\|\eta\|_{\sqrt{K_{I}}}^{2}=\sqrt{\frac{\eta^{2} \wedge \bar{\eta}^{2}}{(2 \mathrm{i})^{n}(-1)^{n(n+1) / 2} \omega^{n} / n !}} .
$$

This defines a Chern connection $\nabla^{\sqrt{K_{I}}}$ on $\sqrt{K_{I}}$ as in Lemma 2.6. The curvature of $\nabla^{\sqrt{K_{I}}}$ is then $F_{\nabla \sqrt{K_{I}}}=\frac{\mathrm{i}}{2} \rho_{I}$, where $\rho_{I}$ is the Ricci form on $X$.

The quantum Hilbert space for the half-form corrected Kähler quantization of $X$ is defined to be

$$
\mathcal{H}_{I}^{Q}:=\left\{s \in \Gamma\left(\ell \otimes \sqrt{K_{I}}\right):\left(\nabla_{\overline{\mathcal{P}}_{I}}^{\ell} \otimes 1+1 \otimes \nabla_{\overline{\mathcal{P}}_{I}}^{\sqrt{K_{I}}}\right) s=0\right\},
$$

where $\mathcal{P}_{I}$ is the holomorphic polarization of $X$ determined by $I$.

Recall from (2.1) that the bundle $\left|\sqrt{K_{I}}\right|$ has a trivializing covariantly constant section

$$
\mu_{I}=\frac{|d Z|^{\frac{1}{2}}}{\|d Z\|_{K_{I}}^{\frac{1}{2}}} .
$$

This defines an isomorphism

$$
\mathcal{H}_{I}^{Q} \cong \mathcal{B}_{I}^{Q} \otimes \mu_{I}
$$

where

$$
\mathcal{B}_{I}^{Q}:=\left\{s \in \Gamma\left(\ell \otimes \widetilde{\sqrt{K}}^{U(1)}\right):\left(\nabla_{\overline{\mathcal{P}}_{I}}^{\ell} \otimes 1+1 \otimes \nabla_{\overline{\mathcal{P}}_{I}^{\sqrt{K}^{U(1)}}}\right) s=0\right\}
$$

Note that, from Lemma 2.9, the unitarization $\ell \otimes \widetilde{\sqrt{K}}^{U(1)}$ is a smooth complex line bundle independent of $I$. In this way, using the $I$-dependent isomorphisms above, we can describe the quantum Hilbert spaces $\mathcal{H}_{I}^{Q}$ through the Hilbert spaces $\mathcal{B}_{I}^{Q}$ which are subspaces of a fixed linear space $\Gamma\left(\ell \otimes \widetilde{\sqrt{K}}^{U(1)}\right)$. 
We will now use this representation to motivate the definition of the halfform corrected quantum Hilbert space in the more general situation when the canonical bundle of $X$ may not admit a square root.

3.2. Corrected quantization. Let $(X, \omega, I)$ be a compact smooth toric Kähler manifold with toric complex structure $I$ and such that $\left[\frac{\omega}{2 \pi}\right]-\frac{1}{2} c_{1}(X)$ is an ample integral cohomology class. Let the moment polytope be

$$
P_{X}=\left\{x \in \mathbb{R}^{n}: \ell_{j}(x)=\nu_{j} \cdot x+\lambda_{j} \geq 0, \quad j=1, \ldots, r\right\},
$$

where we use the freedom of translating the moment polytope to choose the $\left\{\lambda_{j}\right\}_{j=1, \ldots, r}$ to be half-integral and defined as follows. Consider an equivariant complex line bundle $L \cong \mathcal{O}\left(\lambda_{1}^{L} D_{1}+\cdots+\lambda_{r}^{L} D_{r}\right)$ as in (2.18) and with $U(1)$-valued transition functions (2.20) defined by $\left\{\lambda_{j}^{L}\right\}_{j=1, \ldots, r}$, such that $c_{1}(L)=\left[\frac{\omega}{2 \pi}\right]-\frac{1}{2} c_{1}(X)$. As in (2.15), the $\left\{\lambda_{j}^{L}\right\}_{j=1, \cdots, r}$ define a polytope with integral vertices, $P_{L}$. The half-integral $\left\{\lambda_{j}\right\}_{j=1, \ldots, r}$ in (3.6) are then defined by

$$
\lambda_{j}:=\lambda_{j}^{L}+\frac{1}{2} \in \frac{1}{2}+\mathbb{Z}, j=1, \ldots, r,
$$

in accordance with the fact that $\operatorname{div}(d Z)=-D_{1} \cdots-D_{r}$ (see Lemma 2.10). Note that $P_{L}$ is obtained from the moment polytope $P_{X}$ by shifts of $\frac{1}{2}$ along each of the integral primitive inward pointing normals. (See Remarks 3.7 and 3.8 below for examples.) We will call $P_{L} \subset P_{X}$ the corrected polytope.

We equip $L$ with a $U(1)$ connection $\nabla^{I}$ with curvature $F_{\nabla^{I}}=-\mathrm{i} \omega+\frac{\mathrm{i}}{2} \rho_{I}$. Since $H^{1}(X)=0$ this connection is unique up to isomorphism.

Following the reasoning in the last section, and noticing that $\sqrt{\left|K_{I}\right|}$ and $\mu_{I}$ (see (3.4)) exist always even if $\sqrt{K_{I}}$ does not, and that the Hermitian structure on $\sqrt{\left|K_{I}\right|}$ gives $\| \mu_{I}||_{\sqrt{\left|K_{I}\right|}}=1$, we set

Definition 3.1. The quantum Hilbert space for the half-form corrected Kähler quantization of $(X, \omega, L, I)$ is defined by

$$
\mathcal{H}_{I}^{Q}=\mathcal{B}_{I}^{Q} \otimes \mu_{I},
$$

where

$$
\mathcal{B}_{I}^{Q}=\left\{s \in \Gamma(L): \nabla_{\overline{\mathcal{P}}_{I}}^{I} \cdot s=0\right\} .
$$

The inner product is defined by

$$
\left\langle\sigma \otimes \mu_{I}, \sigma^{\prime} \otimes \mu_{I}\right\rangle=\left\langle\sigma, \sigma^{\prime}\right\rangle=\frac{1}{(2 \pi)^{n}} \int_{X} h^{L}\left(\sigma, \sigma^{\prime}\right) \frac{\omega^{n}}{n !} .
$$


Now fix a choice of symplectic potential $g$ for the complex structure $I$ on $X$. We define the connection $\nabla^{I}$ on $L$ by (using Lemma 2.13 and (3.2))

$$
\begin{aligned}
\Theta_{v} & :=\frac{\nabla^{I} \mathbf{1}_{v}^{U(1)}}{\mathbf{1}_{v}^{U(1)}}=-\mathrm{i} x_{v} \cdot d \theta_{v}+\frac{\mathrm{i}}{2} \sum_{k=1}^{n} d \theta_{v}^{k}+\frac{\mathrm{i}}{4}\left(\frac{\partial}{\partial x_{v}} \log \operatorname{det} G_{v}\right) \cdot G_{v}^{-1} d \theta_{v} \\
& =-\mathrm{i} x_{v} \cdot d \theta_{v}+\frac{\mathrm{i}}{2} \operatorname{Im}\left(\partial \log \operatorname{det} G_{v}+\sum_{k=1}^{n} d z_{v}^{k}\right) .
\end{aligned}
$$

On the open orbit $\check{X}$, the connection is then given by

$$
\begin{aligned}
\Theta_{0} & :=-\mathrm{i} x \cdot d \theta+\frac{\mathrm{i}}{4}\left(\frac{\partial}{\partial x} \log \operatorname{det} G\right) \cdot G^{-1} d \theta \\
& =-\mathrm{i} x \cdot d \theta+\frac{\mathrm{i}}{2} \operatorname{Im} \partial \log \operatorname{det} G .
\end{aligned}
$$

One may check that $\Theta_{v}-\Theta_{v^{\prime}}=d \log \tilde{g}_{v^{\prime} v}^{L}$ and $\Theta_{v}-\Theta_{0}=d \log \tilde{g}_{0 v}^{L}$ so that $\left\{\Theta_{0}, \Theta_{v}: v \in V\right\}$ does indeed define a $U(1)$-connection on $L$.

Remark 3.2. Note that, even though $d \theta_{v}^{j}$ is singular as $x_{v}^{j} \rightarrow 0,(3.9)$ defines a non-singular 1-form on $U_{v}$, as can be verified by studying the behavior of $G_{v}$ or using the coordinates $\left\{a_{v}^{j}, b_{v}^{j}\right\}_{j=1, \ldots, n}$.

The complex structure $I$ and the connection $\nabla^{I}$ combine to give a holomorphic structure on $L$ which we can describe by giving the resulting $I$-holomorphic sections of $L$.

Let $h_{0}^{I}(x)=x \cdot \partial g / \partial x-g$ and $h_{v}^{I}\left(x_{v}\right)=x_{v} \cdot \partial g / \partial x_{v}-g$. Also, note that $\operatorname{det} G_{v}=\left(\operatorname{det} A_{v}\right)^{-2} \operatorname{det} G$.

Lemma 3.3. An I-holomorphic section of $L, s \in \mathcal{B}_{I}^{Q}$, is locally given by $\left.s\right|_{U_{v}}=s_{v} \mathbf{1}_{v}^{U(1)}$ where the function $s_{v} \in C^{\infty}\left(U_{v}\right)$ is of the form

$$
F_{v}\left(w_{v}\right) \mathrm{e}^{-h_{v}^{I}\left(x_{v}\right)} \mathrm{e}^{-\mathrm{i} \mathbf{1} / 2 \cdot \theta_{v}}\left\|d Z_{v}\right\|_{K_{I}}^{1 / 2} .
$$

On the orbit one then obtains $\left.s\right|_{U_{0}}=s_{0} \mathbf{1}_{0}^{U(1)}$, where the function $s_{0} \in$ $C^{\infty}\left(U_{0}\right)$ is of the form

$$
F_{0}(w) \mathrm{e}^{-h_{0}^{I}(x)}\|d Z\|_{K_{I}}^{1 / 2},
$$

where $F_{0}$ is holomorphic and $F_{v}\left(w_{v}\right)=w_{v}^{\lambda_{v}} F_{0}\left(w_{v}^{A_{v}}\right)\left|\operatorname{det} A_{v}\right|^{\frac{1}{2}}$.

Remark 3.4. Since the $\lambda_{v}$ are generally only half-integer, the "functions" $F_{v}$ in the above theorem are not single valued. However, $F_{v} \mathrm{e}^{-\mathrm{i} \frac{1}{2} \cdot \theta_{v}}$ is single valued. In fact, the collection $\left\{F_{0}, F_{v}: v \in V\right\}$ defines a ramified section of $L$. There are other possible geometric interpretations of such an object, for instance through the notion of Kawamata covering, but we will not pursue them here. 
Proof. We compute first in the open orbit. From (2.21), we see that

$$
-\mathrm{i} x \cdot d \theta\left(\frac{\partial}{\partial \bar{z}^{j}}\right)=\frac{1}{2} x^{j} .
$$

Recall that $x$ can be expressed in terms of $y$ via the Legendre transform $x=\partial h_{0}^{I} / \partial y$, where $h_{0}^{I}=x \cdot y-g$. Since $\partial / \partial z^{j}=\frac{1}{2}\left(\partial / \partial y^{j}-\mathrm{i} \partial / \partial \theta^{j}\right)$, we have $x^{j}=2 \partial h_{0}^{I} / \partial \bar{z}^{j}$ so that

$$
-\mathrm{i} x \cdot d \theta\left(\frac{\partial}{\partial \bar{z}^{j}}\right)=\frac{\partial h_{0}^{I}}{\partial \bar{z}^{j}} .
$$

Next, from Lemma 2.13 we know that

$$
\begin{aligned}
\frac{\mathrm{i}}{4}\left(\frac{\partial}{\partial x} \log \operatorname{det} G\right) G^{-1} d \theta & =\frac{\mathrm{i}}{2} \operatorname{Im} \partial \log \operatorname{det} G \\
& =\frac{1}{4}(\partial-\bar{\partial}) \log \operatorname{det} G
\end{aligned}
$$

so that

$$
\frac{\mathrm{i}}{4}\left(\frac{\partial}{\partial x} \log \operatorname{det} G\right) G^{-1} d \theta\left(\frac{\partial}{\partial \bar{z}^{j}}\right)=-\frac{1}{4} \frac{\partial}{\partial \bar{z}^{j}} \log \operatorname{det} G .
$$

Combining (3.13) and (3.14), we see that a section $s=f \mathbf{1}_{0}^{U(1)} \in \Gamma_{U_{0}}(L)$ is holomorphic if and only if $f$ satisfies the differential equation

$$
\frac{\partial f}{\partial \bar{z}^{j}}+f \frac{\partial}{\partial \bar{z}^{j}}\left(h_{0}^{I}-\frac{1}{4} \log \operatorname{det} G\right)=0
$$

for each $j=1, \ldots, n$. We solve this easily to see that $s$ is holomorphic if and only if $f$ is of the form

$$
f=F(w) \mathrm{e}^{-h_{0}^{I}}(\operatorname{det} G)^{1 / 4},
$$

where $F$ is an holomorphic function in $U_{0}$. From Lemma 2.12, we recognize $(\operatorname{det} G)^{1 / 4}=\|d Z\|_{K}^{1 / 2}$ to obtain $(3.12)$ as desired.

We have computed that $F(w) \mathrm{e}^{-h_{0}^{I}}(\operatorname{det} G)^{1 / 4} \mathbf{1}_{0}^{U(1)}$ is holomorphic. Using the transition functions $\tilde{g}_{v 0}$, we conclude that $F(w) \mathrm{e}^{-h_{0}^{I}}(\operatorname{det} G)^{1 / 4} \tilde{g}_{v 0}\left(w_{v}\right)$ $\mathbf{1}_{v}^{U(1)}$, when expressed in terms of $w_{v}$, should be holomorphic. First, note that

$$
x \cdot y=x_{v} \cdot y_{v}-\lambda_{v} \cdot y_{v},
$$

which implies $h_{0}^{I}=h_{v}^{I}-\lambda_{v} \cdot y_{v}$. From (2.17) we therefore see that the holomorphic combination in $U_{v}$ should be

$$
\begin{aligned}
& F(w) \mathrm{e}^{-h_{v}^{I}+\lambda_{v} \cdot y_{v}}(\operatorname{det} G)^{1 / 4} \mathrm{e}^{\mathrm{i} \lambda_{v}^{L} \cdot \theta_{v}} \mathbf{1}_{v}^{U(1)} \\
& \quad=F(w) \mathrm{e}^{-h_{v}^{I}} \mathrm{e}^{\lambda_{v} \cdot y_{v}+\mathrm{i} \lambda_{v} \cdot \theta_{v}} \mathrm{e}^{-\mathrm{i} \mathbf{1} / 2 \cdot \theta_{v}}(\operatorname{det} G)^{1 / 4} \mathbf{1}_{v}^{U(1)} \\
& \quad=F(w) w_{v}^{\lambda_{v}} \mathrm{e}^{-h_{v}^{I}} \mathrm{e}^{-\mathrm{i} \mathbf{1} / 2 \cdot \theta_{v}}\left|\operatorname{det} A_{v}\right|^{\frac{1}{2}}\left\|d Z_{v}\right\|_{K_{I}}^{1 / 2} \mathbf{1}_{v}^{U(1)} .
\end{aligned}
$$


By $(2.12)$ we see that if we set $F_{v}\left(w_{v}\right):=F\left(w_{v}^{A_{v}}\right) w_{v}^{\lambda_{v}}\left|\operatorname{det} A_{v}\right|^{\frac{1}{2}}$, we obtain (3.11) and the final statement of the lemma as desired.

Remark 3.5. We can rewrite a local holomorphic section on $U_{v} \cap U_{0}$ in a way similar to that in [BFMN11] as follows:

$$
\begin{aligned}
& F_{v}\left(w_{v}\right) \mathrm{e}^{-h_{v}^{I}\left(x_{v}\right)} \mathrm{e}^{-1 / 2 \cdot \theta_{v}}\left\|d Z_{v}\right\|_{K_{I}}^{1 / 2} \\
& \quad=F_{0}\left(w_{v}^{A_{v}}\right) w_{v}^{\lambda_{v}} \mathrm{e}^{-h_{v}^{I}\left(x_{v}\right)} \mathrm{e}^{-\mathrm{i} \mathbf{1} / 2 \cdot \theta_{v}}\left|\operatorname{det} A_{v}\right|^{\frac{1}{2}}\left\|d Z_{v}\right\|_{K_{I}}^{1 / 2} \\
& \quad=F_{0} \mathrm{e}^{-h_{v}^{I}+\lambda_{v} \cdot y_{v}} \mathrm{e}^{\mathrm{i} \lambda_{v}^{L} \cdot \theta_{v}}\left|\operatorname{det} A_{v}\right|^{\frac{1}{2}}\left\|d Z_{v}\right\|_{K_{I}}^{1 / 2} .
\end{aligned}
$$

The combination $h_{v}^{I}-\lambda_{v} \cdot y_{v}$ corresponds to $h_{m}($ for $m=v)$ in [BFMN11].

Theorem 3.6. The Hilbert space $\mathcal{B}_{I}^{Q}$ of holomorphic sections of L has an orthogonal basis $\left\{\sigma^{m}\right\}_{m \in P_{L} \cap \mathbb{Z}^{n}}$ where $\sigma^{m}$ is locally given by

$$
\begin{aligned}
& \sigma_{0}^{m}=w^{m} \mathrm{e}^{-h_{0}^{I}}\|d Z\|_{K_{I}}^{1 / 2} \mathbf{1}_{0}^{U(1)}, \quad \text { and } \\
& \sigma_{v}^{m}=w_{v}^{A_{v} m+\lambda_{v}^{L}} \mathrm{e}^{-h_{v}^{I}+\mathbf{1} / 2 \cdot y_{v}}\left|\operatorname{det} A_{v}\right|^{\frac{1}{2}}\left\|d Z_{v}\right\|_{K_{I}}^{1 / 2} \mathbf{1}_{v}^{U(1)},
\end{aligned}
$$

over the open orbit and holomorphic vertex charts, respectively. The corresponding orthogonal basis for the quantum Hilbert space $\mathcal{H}_{I}^{Q}$ is given by $\left\{\hat{\sigma}^{m}:=\sigma^{m} \otimes \mu_{I}\right\}_{m \in P_{L} \cap \mathbb{Z}^{n}}$.

Proof. From Lemma 3.3 we can certainly find a basis for the space of holomorphic sections of $L$ consisting of elements given locally over the open orbit by

$$
w^{m} \mathrm{e}^{-h_{0}^{I}}\|d Z\|_{K_{I}}^{1 / 2} \mathbf{1}_{0}^{U(1)},
$$

where $m \in \mathbb{Z}^{n}$, with the corresponding expressions over the holomorphic vertex charts. Such a section will have poles unless $m$ belongs to the corrected polytope $P_{L}$. The fact that $\sigma^{m}$ and $\sigma^{m^{\prime}}$ are orthogonal for $m \neq m^{\prime}$ follows immediately from integration along $\mathbb{T}^{n}$.

Therefore, the space of half-form corrected holomorphic wave functions for the Kähler quantization of $X$ has a natural basis whose elements are labeled by the integral points of the corrected polytope $P_{L}$. These coincide also with the (interior) integral points of the moment polytope $P_{X}$ and they correspond to shifted non-singular Bohr-Sommerfeld fibers of $(X, \omega)$.

Remark 3.7. Pictured below is the moment polytope $P_{X}$ for $X=\mathbb{C P}^{1}$, in the case $\left[\frac{\omega}{2 \pi}\right]=\frac{3}{2} c_{1}\left(\mathbb{C P}^{1}\right)=3 c_{1}(\mathcal{O}(1))$. On the left, we show the more standard choice of moment polytope, with integral vertices. On the right, we show the moment polytope chosen in accordance with (3.6) and (3.7), that is such that $\lambda_{1}, \lambda_{2}$ are half-integral. In this example, $L \cong \mathcal{O}(2)$, the corrected polytope is $P_{L}=[0,2]$ and the moment polytope is $P_{X}=\left[-\frac{1}{2}, \frac{3}{2}\right]$. One has $\operatorname{dim} \mathcal{H}^{Q}=3$. 

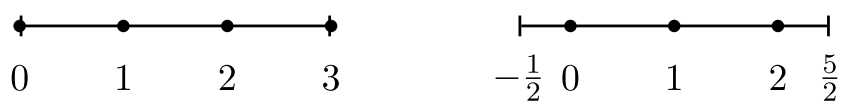

Remark 3.8. According to Theorem 3.6, we can count holomorphic sections of $L$ by counting integral points inside the moment polytope $P_{X}$, which are exactly the integral points which occur in the corrected polytope $P_{L}$. Pictured below is one of such polytopes when $X=\mathbb{C P}^{2} \# \overline{\mathbb{C P}}^{2}$, that is, $\mathbb{C P}^{2}$ blown up at a point.

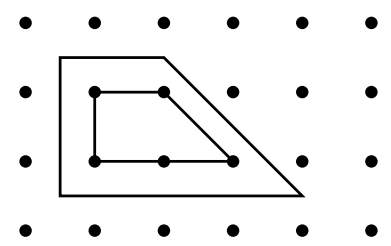

Remark 3.9. One can consider the case $[\omega / 2 \pi]=c_{1}(X) / 2$, where $L=\mathcal{O}_{X}$ is just the structure sheaf of $X$ (which is, of course, not ample). In this case, there is only one integral point inside the polytope $P_{X}$ corresponding to the constant function $1 \in H^{0}\left(\mathcal{O}_{X}\right)$. As we will see in Section 4.3 , when we study degenerations of the complex structure, also in this case we have convergence to a Dirac delta distribution supported on the (shifted) Bohr-Sommerfeld fiber above that integral point.

\section{Half-form corrected quantization in the singular real toric polarization}

4.1. Distributional sections. In order to study quantization in the real toric polarization of $(X, \omega)$, following [BFMN11], we consider distributional sections of $L$. Let us briefly recall how one can define covariant differentiation in this case. Let $L^{2}(L)$ denote the Hilbert space of $L^{2}$ sections of $L$. Consider the rigged Hilbert space (see Sections 4.2 and 4.3 of $[\mathbf{G V}]$ ) $\left(\Gamma(L), L^{2}(L), \Gamma(\bar{L})^{\prime}\right)$, where $\Gamma(\bar{L})^{\prime}$ is the space of distributional sections of $L$ given by the topological dual of $\Gamma(\bar{L})$. One has the continuous inclusions

$$
\Gamma(L) \subset L^{2}(L) \subset \Gamma(\bar{L})^{\prime},
$$

where we embed $\sigma \in \Gamma(L) \mapsto i(\sigma) \in \Gamma(\bar{L})^{\prime}$ via the Liouville volume form; i.e.,

$$
i(\sigma)(\bar{\tau}):=\frac{1}{(2 \pi)^{n}} \int_{X} h^{L}(\sigma, \tau) \frac{\omega^{n}}{n !} .
$$

(In particular, we may view $I$-holomorphic sections of $L$ as distributional sections.) We have then, for $\sigma \in \Gamma(L)$,

$$
i(\sigma)(\bar{\tau})=\langle\sigma, \tau\rangle_{L}, \quad \forall \tau \in \Gamma(L) .
$$


Let $\nabla^{L}$ be a connection on $L$ and let $\nabla^{*}$ be the adjoint of the (unbounded) operator $\nabla^{L}$ on the Hilbert space $L^{2}(X, L)$, so that

$$
i\left(\nabla^{L} \sigma\right)(\bar{\tau})=\left\langle\nabla^{L} \sigma, \tau\right\rangle_{L}=\left\langle\sigma, \nabla^{*} \tau\right\rangle_{L}, \quad \forall \sigma, \tau \in \Gamma(L) .
$$

We can now define covariant differentiation of distributional sections, which we will still denote by $\nabla^{L}$, by

$$
\nabla^{L}(\sigma)(\bar{\tau})=\sigma\left(\overline{\nabla^{*} \tau}\right), \quad \sigma \in \Gamma(\bar{L})^{\prime}, \quad \tau \in \Gamma(L)
$$

so that, as distributions

$$
\nabla^{L}(i \sigma)=i\left(\nabla^{L} \sigma\right), \quad \forall \sigma \in \Gamma(L) .
$$

In the next sections, we will interpret holomorphic sections as distributional sections in this way, and we will identify $\sigma \in \Gamma(L)$ with $\mathrm{i}(\sigma) \in \Gamma(\bar{L})^{\prime}$.

4.2. Quantization in the (singular) real polarization. Recall that the real singular toric polarization is defined by

$$
\mathcal{P}_{\mathbb{R}}(p)=\operatorname{span}_{\mathbb{C}}\left\{\left(\frac{\partial}{\partial \theta^{i}}\right)_{p}, i=1, \ldots, n\right\}, \quad \forall p \in X .
$$

In this section, we will define the half-form corrected quantization of $X$ in this polarization directly in terms of covariantly constant sections. Recall the families of toric complex structures considered in [BFMN11]. For any smooth function $\psi$ which is strictly convex on a neighborhood of $P_{X}$, for any $\varphi \in C_{P_{X}}^{\infty}\left(P_{X}\right)$ and for any $s \in \mathbb{R}_{\geq 0}$, the sum $\varphi+s \psi$ is in $C_{P_{X}}^{\infty}\left(P_{X}\right)$ and hence defines a Kähler structure on $X$ with symplectic potential

$$
g_{s}:=g_{P_{X}}+\varphi+s \psi .
$$

Denote the corresponding $s$-dependent complex structure by $I_{s}$.

If $\mathcal{P}$ is a polarization of $(X, \omega)$, denote by $C^{\infty}(\mathcal{P})$ its space of smooth sections,

$$
C^{\infty}(\mathcal{P})=\left\{\xi \in C^{\infty}(T X \otimes \mathbb{C}): \xi(p) \in \mathcal{P}_{p}\right\},
$$

where $C^{\infty}(T X \otimes \mathbb{C})$ is the space of smooth sections of the complexified tangent bundle of $X$.

Theorem 4.1 ([BFMN11], Theorem 1.2 p. 415, Theorem 3.4, p. 429). Pointwise on the dense open orbit $\check{X}$, as vector fields,

$$
\frac{\partial}{\partial \bar{z}_{s}^{j}}=\frac{1}{2}\left(\frac{\partial}{\partial y_{s}^{j}}+\mathrm{i} \frac{\partial}{\partial \theta^{j}}\right) \rightarrow \frac{\mathrm{i}}{2} \frac{\partial}{\partial \theta^{j}}, \quad \text { as } s \rightarrow \infty .
$$

Therefore, at each point $p \in \check{X}$, the holomorphic polarizations $\mathcal{P}_{s}, s \geq 0$ of $X$, associated to the complex structures $I_{s}$, converge, as $s \rightarrow \infty$, to the real toric polarization, in the Lagrangian Grassmannian of $T_{p} X \otimes \mathbb{C}$ and

$$
C^{\infty}\left(\lim _{s \rightarrow \infty} \mathcal{P}_{s}\right)=C^{\infty}\left(\mathcal{P}_{\mathbb{R}}\right)
$$


Remark 4.2. The equality in (4.1) is an equality of spaces of smooth sections of two different polarizations $\lim _{s \rightarrow \infty} \mathcal{P}_{s}$ and $\mathcal{P}_{\mathbb{R}}$ which coincide over $\check{X}$ but not over $X \backslash \check{X}$. (See Theorem 3.4 of [BFMN11]).

From the expressions for the half-form corrected connection in (3.9), we see that in the local trivializations $\mathbf{1}_{v}^{U(1)}$

$$
-2 \mathrm{i} \nabla_{\partial / \partial \bar{z}_{s v}^{j}}^{I_{s}} \rightarrow \nabla_{\partial / \partial \theta_{v}^{j}}^{\mathbb{R}}:=\frac{\partial}{\partial \theta_{v}^{j}}-\mathrm{i} x_{v}^{j}+\frac{\mathrm{i}}{2}, \quad \text { as } s \rightarrow \infty,
$$

in the sense that

$$
-2 \mathrm{i}\left(\nabla_{\partial / \partial \bar{z}_{s_{v}}^{j}}^{I_{s}} \sigma\right)(\bar{\tau}) \rightarrow\left(\nabla_{\partial / \partial \theta_{v}^{j}}^{\mathbb{R}} \sigma\right)(\tau), \quad \text { as } s \rightarrow \infty,
$$

$\forall \sigma \in \Gamma(\bar{L})^{\prime}, \forall \tau \in \Gamma(L)$. Similarly, from (3.10), on the open orbit in the trivialization $\mathbf{1}_{0}^{U(1)}$ we have

$$
-2 \mathrm{i} \nabla_{\partial / \partial \bar{z}_{s}^{j}}^{I_{s}} \rightarrow \nabla_{\partial / \partial \theta^{j}}^{\mathbb{R}}=\frac{\partial}{\partial \theta^{j}}-\mathrm{i} x^{j}, \quad \text { as } s \rightarrow \infty .
$$

We will take the expressions on the right-hand side of (4.2) and (4.3) to define a partial connection $\nabla^{\mathbb{R}}$ on $\Gamma(\bar{L})^{\prime}$, along $\mathcal{P}_{\mathbb{R}}$, which will be used to define the quantization in this polarization. Let

$$
\mathcal{B}_{\mathbb{R}}^{Q}=\operatorname{ker} \nabla^{\mathbb{R}}=\bigcap_{j=1}^{n} \operatorname{ker} \nabla_{\partial / \partial \theta^{j}}^{\mathbb{R}} \subset \Gamma(\bar{L})^{\prime} .
$$

Remark 4.3. We note that additive term $\frac{\mathrm{i}}{2}$ in the right-hand side of (4.2), corresponds to a limiting Chern connection on $K_{I_{s}}$ which is flat on $U_{0}$ and singular along $\cup_{i=1}^{r} D_{i}$. The addition of this singular connection to the prequantum connection is at the core of our approach to the half-form quantization in the singular real toric polarization. We will describe in more detail the singular behavior of the limiting connection at the end of this section.

Remark 4.4. As explained in the previous section, we should think of $L$ as the tensor product of the uncorrected bundle $\ell$ with the smooth bundle with $U(1)$-valued transition functions $\widetilde{\sqrt{K}}^{U(1)}$, though in general these may not exist individually. On the other hand, the geometric quantization associated to a polarization $\mathcal{P}$ is supposed to be the space of $\mathcal{P}$-covariantly constant sections of the tensor product of the uncorrected bundle with the square root of the canonical bundle associated $\mathcal{P}$. For the real polarization $\mathcal{P}_{\mathbb{R}}$, the sections of the associated canonical bundle are $n$-forms of the form $a(x) d x^{1} \wedge$ $\cdots \wedge d x^{n}$. Of course, the $U(1)$-part of this is hidden in the sections of $L$, and what is missing is the modulus of the square root of the canonical bundle associated to $\mathcal{P}_{\mathbb{R}}$.

To put it another way, according to the standard procedures of geometric quantization, we should actually define the quantization $\mathcal{H}_{\mathbb{R}}^{Q}$ to be sections 
of $L \otimes \sqrt{\left|K_{\mathcal{P}_{\mathbb{R}}}\right|}$. Some care must be taken to interpret exactly what is meant by $\left|K_{\mathcal{P}_{\mathbb{R}}}\right|$ (and hence what is meant by its square root). In the next section, we will see that sections of $\sqrt{\left|K_{\mathcal{P}_{\mathbb{R}}}\right|}$ can be thought of as maps on the space of $n$-tuples of vector fields. Then, $\sqrt{\left|K_{\mathcal{P}_{\mathbb{R}}}\right|}$ admits a canonical section $d X:=$ $d x^{1} \wedge \cdots \wedge d x^{n}$ on $U_{0}$, and 0 otherwise. Hence, we should define $\mathcal{H}_{\mathbb{R}}^{Q}=$ $\mathcal{B}_{\mathbb{R}}^{Q} \otimes \sqrt{|d X|}$. Of course, at this point, such a change is merely cosmetic. On the other hand, we will see in the next section that such expressions arise naturally when studying the degenerations of the complex structure on $X$ to the real polarization at the level of holomorphic sections.

Definition 4.5. The vector space of quantum states for the half-form corrected quantization of $(X, \omega, L)$ in the toric polarization $\mathcal{P}_{\mathbb{R}}$ is defined by

$$
\mathcal{H}_{\mathbb{R}}^{Q}=\mathcal{B}_{\mathbb{R}}^{Q} \otimes \sqrt{|d X|},
$$

where $\mathcal{B}_{\mathbb{R}}^{Q}$ was defined in (4.4).

Remark 4.6. A natural Hilbert space structure in $\mathcal{H}_{\mathbb{R}}^{Q}$ will be introduced in Section 4.3, via degeneration of Kähler quantizations of $(X, \omega, L, I)$.

The open orbit $U_{0}$ carries a free $\mathbb{T}^{n}$-action, which lifts to $L_{U_{0}}$ via geometric quantization. This action is generated by

$$
\nabla_{\frac{\partial}{\partial \theta}}+\mathrm{i} x
$$

Then, the trivializing section $\mathbf{1}_{0}^{U(1)}$ is $\mathbb{T}^{n}$-invariant and if $\tau \in \Gamma_{U_{0}}(L)$ is given by $\tau=\tau_{0} \mathbf{1}_{0}^{U(1)}$ for a smooth function $\tau_{0} \in C^{\infty}\left(U_{0}\right)$, we can decompose it into Fourier modes with respect to the $\mathbb{T}^{n}$-action. Specifically,

$$
\tau_{0}(x, \theta)=\sum_{m \in \mathbb{Z}^{n}} \mathrm{e}^{-\mathrm{i} m \cdot \theta} \hat{\tau}_{0, m}(x),
$$

where $\hat{\tau}_{0, m}(x)=\frac{1}{(2 \pi)^{n}} \int_{\mathbb{T}^{n}} \mathrm{e}^{\mathrm{i} m \cdot \theta} \tau_{0}(x, \theta) d \theta$ is the $m$ th Fourier mode of $\tau_{0}$.

For $m \in P_{L} \cap \mathbb{Z}^{n}$, let $\delta^{m} \in \Gamma(\bar{L})^{\prime}$ be the distributional section defined by

$$
\delta^{m}(\bar{\tau})=\overline{\hat{\tau}}_{m}(m)=\frac{1}{(2 \pi)^{n}} \int_{\mathbb{T}^{n}} \mathrm{e}^{\mathrm{i} m \cdot \theta} \bar{\tau}_{0}(m, \theta) d \theta,
$$

for all $\tau \in \Gamma(L)$. We have

Theorem 4.7. The vector space $\mathcal{H}_{\mathbb{R}}^{Q}$ is the finite-dimensional vector space generated by $\left\{\delta^{m} \otimes \sqrt{|d X|}\right\}_{m \in P_{X} \cap \mathbb{Z}^{n}}$.

Therefore, quantization in the real polarization is also given by the integral points in the interior of the moment polytope. Recall, from Section 3.2 and Remarks 3.7 and 3.8 , that $P_{L} \subset P_{X}$ is obtained from $P_{X}$ by a one-half shift along the inward pointing normals to the facets of $P_{X}$. In Section 4.3, these distributional sections will be described as coming from holomorphic sections through degeneration of the complex structure. 
Proof of Theorem 4.7. By acting with $\nabla_{\partial / \partial \theta^{j}}^{\mathbb{R}}, j=1, \ldots, n$, in (4.3) on the distributions $\delta^{m}$ in (4.5) we conclude that they belong to $\operatorname{ker} \nabla^{\mathbb{R}}$. Moreover, any element of the kernel can be restricted to the open orbit, by restricting it to sections of $\bar{L}$ with compact support contained in the open orbit. From, Proposition 3.1 in [BFMN11], we see that such restrictions can only have support along $\mu^{-1}\left(\check{P}_{X} \cap \mathbb{Z}^{n}\right)$, as these are the only fibers along which $\nabla^{\mathbb{R}}$ has trivial holonomy, and one easily verifies that $\delta^{m}$ is the unique (up to a constant) solution supported on $\mu^{-1}(m)$.

It remains to be shown that there are no more elements in the kernel of $\nabla^{\mathbb{R}}$. All we need to show is that there are no solutions with support along $\mu^{-1}\left(\partial P_{X}\right)$. Let us consider a solution with support along $\mu^{-1}\left(x_{v}^{j}=0\right)$, for some fixed $j=1, \ldots, n$. Let $\check{x}_{v}=\left(x_{v}^{1}, \ldots, x_{v}^{j-1}, x_{v}^{j+1}, \ldots, x_{v}^{n}\right)$ and $\check{\theta}_{v}=$ $\left(\theta_{v}^{1}, \ldots, \theta_{v}^{j-1}, \theta_{v}^{j+1}, \ldots, \theta_{v}^{n}\right)$. In a neighborhood of the preimage by $\mu$ of the interior of the facet $x_{v}^{j}=0$ of $P_{X}$, we can take coordinates $\left(u, v, \check{x}_{v}, \check{\theta}_{v}\right)$ (see, for example [DP09, BFMN11]), so that $x_{v}^{j}=0 \Leftrightarrow(u, v)=(0,0)$ and

$$
\nabla_{\frac{\partial}{\partial \theta_{v}^{j}}}^{\mathbb{R}}=-\mathrm{i}\left(-v \frac{\partial}{\partial u}+u \frac{\partial}{\partial v}\right)+\frac{\mathrm{i}}{2},
$$

in that neighborhood. A solution with support along the facet will be of the form,

$$
\sigma=\sum_{k, l=0}^{\infty} \alpha_{k l}\left(\check{x}_{v}, \check{\theta}_{v}\right) \delta^{(k)}(u) \delta^{(l)}(v) \mathbf{1}_{v}^{U(1)},
$$

where only a finite number of terms in the sum can be non-zero and where $\delta^{(k)}$ denotes the order- $k$ derivative of the Dirac $\delta$ distribution. (See Theorem 2.3.5 of [Hör90].) Using $x \delta^{(k)}(x)=-k \delta^{(k-1)}(x)$ and polynomial test sections of the form $u^{k} v^{l} \chi \mathbf{1}_{v}^{U(1)}$, where $\chi$ is a cutoff function, which is constant

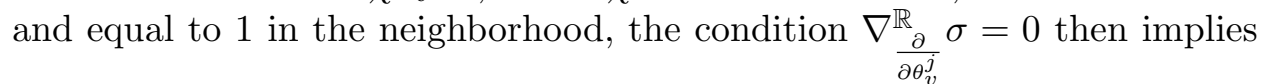
that such a distributional section is zero. Therefore, no non-zero solutions of this form exist.

As we will see in the next section, there is complete agreement between the direct approach to half-form corrected quantization in the real polarization and the approach based on degeneration of holomorphic sections. We note that the partial connection $\nabla^{\mathbb{R}}$ "remembers" the degeneration procedure due to the contribution of the Ricci-curvature term.

It is possible to gain some more geometric intuition about the fact that the boundary of $P_{X}$ does not contribute to the kernel of $\nabla^{\mathbb{R}}$, unlike what happens without the half-form correction [BFMN11]. As $s \rightarrow \infty$, the piece of the connection $\nabla^{I_{s}}$ coming from the Levi-Civita connection on the canonical bundle of $X$ develops curvature singularities outside of the open orbit $U_{0}$. This is behind the fact, shown above, that for the real polarization the 
half-form correction forbids solutions of the covariant constancy equations supported on $\mu^{-1}\left(\partial P_{X}\right)$. For completeness, let us describe these curvature singularities in more detail.

Recall Lemma 2.13, which gives explicit expressions for the Chern connection 1-forms on $K^{U(1)}$ (induced from the Chern connection on $K$ ) in the vertex atlas. The proof of the following Proposition is immediate.

\section{Proposition 4.8.}

$$
\lim _{s \rightarrow \infty} \Theta_{v}^{K_{I_{s}}}=\sum_{j=1}^{n} d \theta_{v}^{j},
$$

in the sense that for any closed curve $C$, with $C \subset U_{v} \backslash \mu^{-1}\left(\partial P_{X}\right)=U_{0} \cong$ $\left(\mathbb{C}^{*}\right)^{n}$, the holonomy of the singular connection, along $C$ depends only on the homotopy class of $C$ in $U_{0}$ and,

$$
\lim _{s \rightarrow \infty} \oint_{C} \Theta_{v}^{K_{I_{s}}}=\mathrm{i} \oint_{C} \sum_{j=1}^{n} d \theta_{v}^{j} .
$$

Therefore, in the limit $s \rightarrow \infty$, we obtain a singular connection on $K^{U(1)}$, flat on $\mu^{-1}\left(\check{P}_{X}\right)$, with curvature supported on $\mu^{-1}\left(\partial P_{X}\right)=\cup_{i=1}^{r} D_{i}$ and with non-vanishing monodromies around the toric invariant divisors. In the vertex chart $U_{v}$ the curvature, in the limit $s \rightarrow \infty$, is given by the following current:

$$
2 \pi \mathrm{i} \sum_{j=1}^{n} \delta\left(a_{v}^{j}\right) \delta\left(b_{v}^{j}\right) d a_{v}^{j} \wedge d b_{v}^{j},
$$

where $\left(a_{v}^{1}, b_{v}^{1}, \ldots, a_{v}^{n}, b_{v}^{n}\right)$, with $a_{v}^{j}=\sqrt{x_{v}^{j}} \cos \theta_{v}^{j}, b_{v}^{j}=\sqrt{x_{v}^{j}} \sin \theta_{v}^{j}, i=$ $1, \ldots, n$, are coordinates on $U_{v}$.

4.3. Degeneration to the real polarization. In this section, we will obtain the degeneration, as $s \rightarrow \infty$, of the (appropriately $L^{2}$-normalized) elements $\hat{\sigma}_{s}^{m}$ of the orthogonal basis of $\mathcal{H}_{I_{s}}^{Q}$, defined in Theorem 3.6, to the same distributional sections $\delta^{m} \otimes \sqrt{|d X|} \in \mathcal{H}_{\mathbb{R}}^{Q}$ obtained in the previous section, see Definition 4.5, (4.5) and Theorem 4.7. In particular, this will allow us to define a natural inner product in $\mathcal{H}_{\mathbb{R}}^{Q}$. We will first study the degeneration of the basis elements $\sigma_{s}^{m}$ of $\mathcal{B}_{I_{s}}^{Q}$ and then the degeneration of the sections $\mu_{I_{s}}$ of $\sqrt{\left|K_{I_{s}}\right|}$.

As the complex structure $I_{s}$ varies with $s$, we can regard the spaces $\mathcal{B}_{I_{s}}^{Q}$ of $I_{s}$-holomorphic sections, described in Definition 3.1, as finite-dimensional subspaces of the fixed infinite-dimensional space of distributional sections of $L$ (see Section 4.1). That is, for all $s$,

$$
\mathcal{B}_{I_{s}}^{Q} \subset \Gamma(L) \subset \Gamma(\bar{L})^{\prime} .
$$


We will study (weak) convergence of $I_{s}$-holomorphic sections in $\Gamma(\bar{L})^{\prime}$, as $s \rightarrow$ $\infty$. Denote the $I_{s}$-holomorphic section of $L$ associated to $m \in P_{L} \cap \mathbb{Z}^{n}$ by $\sigma_{s}^{m}$, as in Theorem 3.6. Suppose $\tau \in \Gamma(L)$ is given locally by $\left\{\tau_{0} \mathbf{1}_{0}^{U(1)}, \tau_{v} \mathbf{1}_{v}^{U(1)}\right.$ : $v \in V\}$ and let $m \in P_{L} \cap \mathbb{Z}^{n}$. Then since $\check{X}$ and the holomorphic vertex charts $U_{v}$ are dense in $X$, we see from Theorem 3.6 that

$$
\begin{aligned}
i\left(\sigma_{s}^{m}\right)(\bar{\tau}) & =\frac{1}{(2 \pi)^{n}} \int_{\check{X}} w^{m} \mathrm{e}^{-h_{0}^{I_{s}}}\|d Z\|_{K}^{1 / 2} \bar{\tau}_{0} \frac{\omega^{n}}{n !} \\
& =\frac{1}{(2 \pi)^{n}} \int_{U_{v}} w_{v}^{\ell_{v}(m) \cdot y_{s_{v}}-h_{v}^{I_{S}}+\mathbf{1} / 2 \cdot y_{s_{v}}}\left|\operatorname{det} A_{v}\right|^{\frac{1}{2}}\left\|d Z_{v}\right\|_{K}^{1 / 2} \bar{\tau}_{v} \frac{\omega^{n}}{n !}
\end{aligned}
$$

where $y_{s_{v}}=\partial g_{s} / \partial x_{v}$.

The next lemma, which we recall from [BFMN11], will allow us to use Laplace's approximation to compute the asymptotics that we are interested in.

Lemma 4.9. [BFMN11, Lemma 5.1] For $m \in P_{X}$ and any smooth function $\psi$ which is strictly convex on a neighborhood of $P_{X}$, let

$$
f_{m}:=(x-m) \cdot \partial \psi / \partial x-\psi .
$$

Then $f_{m}$ has a minimum value of $-\psi(m)$ on $P$, which is obtained at the unique point $x=m$. Moreover,

$$
\left(\operatorname{Hess} f_{m}\right)(m)=(\operatorname{Hess} \psi)(m) .
$$

Remark 4.10. It is important to observe that the function $f_{m}$ in Lemma 4.9 has a unique minimum on the entire polytope $P_{X}$, not just the interior, which implies that the leading order asymptotics that we will be interested in all arise from the behavior of the integrand at $x=m$.

Recall,

Lemma 4.11 (Laplace's approximation). Suppose a function $f \in C^{2}(R)$ on the closed region $R \subset \mathbb{R}^{n}$ has a unique non-degenerate minimum the unique point $x_{0} \in \check{R}$ in the interior of $R$; so in particular, $\operatorname{Hess}_{x_{0}} f$ is positive definite. Then if $g_{s}$ is a continuous function on $R$ such that $g_{s} \sim s^{r} g_{0}+$ $O\left(s^{r-1}\right), s \rightarrow \infty$, we have

$$
\int_{R} \mathrm{e}^{-s f} g_{s} d x \sim\left(\frac{2 \pi}{s}\right)^{n / 2} \frac{\mathrm{e}^{-s f\left(x_{0}\right)} s^{r} g_{0}\left(x_{0}\right)}{\sqrt{\operatorname{det}(\operatorname{Hess} f)\left(x_{0}\right)}}, \quad s \rightarrow \infty .
$$

Lemma 4.12. As $s \rightarrow \infty$, the leading order asymptotic value of the $L^{2}$-norm of the family of sections $\sigma_{s}^{m}, s \in \mathbb{R}_{>0}$ is

$$
\left\|\sigma_{s}^{m}\right\|_{L^{2}}^{2} \sim \pi^{n / 2} \mathrm{e}^{2 g_{s}(m)} .
$$


Proof. To compute the asymptotics we can restrict the integral to the open orbit, where we have

$$
\begin{aligned}
\left\|\sigma_{s}^{m}\right\|_{L^{2}}^{2} & =\frac{1}{(2 \pi)^{n}} \int_{\check{P}_{X} \times \mathbb{T}^{n}}\left|w^{m}\right|^{2} \mathrm{e}^{-2 h_{0}^{I_{s}}}\|d Z\|_{K} \frac{\omega^{n}}{n !} \\
& =\frac{1}{(2 \pi)^{n}} \int_{\check{P}_{X} \times \mathbb{T}^{n}} \mathrm{e}^{2 m \cdot y_{s}-2\left(x \cdot y_{s}-g_{s}\right)}\left(\operatorname{det} G_{s}\right)^{1 / 2} \frac{\omega^{n}}{n !} \\
& =\int_{\check{P}_{X}} \mathrm{e}^{-2 s((x-m) \cdot \partial \psi / \partial x-\psi)} \mathrm{e}^{-2\left((x-m) \cdot y_{0}-g_{0}\right)}\left(\operatorname{det} G_{s}\right)^{1 / 2} d x,
\end{aligned}
$$

where in the last line we used the fact that $y_{s}=\partial g_{s} / \partial x=\partial g_{0} / \partial x+s \partial \psi / \partial x$. Note that $G_{s}=G_{0}+s$ Hess $\psi$ implies

$$
\operatorname{det} G_{s} \sim s^{n} \operatorname{det} \operatorname{Hess} \psi+O\left(s^{n-1}\right) .
$$

We would like to apply Laplace's approximation to the integral (4.7). By Lemma 4.9, the first exponential has the correct behavior. The only remaining subtlety is to show that the remainder of the integrand is continuous on $P_{X}$, which is not immediate since $g_{s}$ is singular along $\partial P_{X}$. Using the explicit expression (2.6) and the regularity conditions (2.7), we conclude that $\mathrm{e}^{-2\left((x-m) \cdot y_{0}-g_{0}\right)}\left(\operatorname{det} G_{s}\right)^{1 / 2}$ behaves like

$$
\Pi_{i=1}^{r} \ell_{i}(x)^{\left(\frac{1}{2} \ell_{i}(m)-\frac{1}{2}\right)}
$$

times a smooth function on $P_{X}$. Therefore, it is continuous and goes to zero at the boundary of $P_{X}$ precisely when $m$ belongs to the corrected polytope $P_{L} \subset P_{X}$. From another point of view, the integrand in (4.7) is the pointwise norm of the $\mathbb{T}^{n}$-invariant holomorphic section $\sigma_{s}^{m}$, which is necessarily continuous on $P_{X}$. Then, Laplace's approximation (Lemma 4.11) yields

$$
\left\|\sigma_{s}^{m}\right\|_{L^{2}}^{2} \sim\left(\frac{2 \pi}{s}\right)^{n / 2} \frac{\mathrm{e}^{2 s \psi(m)} \mathrm{e}^{2 g_{0}(m)} s^{n / 2} \sqrt{\operatorname{det} \operatorname{Hess} \psi(m)}}{\sqrt{2^{n} \operatorname{det} \operatorname{Hess} \psi(m)}}=\pi^{n / 2} \mathrm{e}^{2 g_{s}(m)}
$$

as desired.

Recall that $G_{s}=G_{0}+s \operatorname{Hess} \psi$, where $G_{0}=\operatorname{Hess}\left(g_{P}+\varphi\right)$.

Theorem 4.13. For each $\tau \in \Gamma(L)$, the leading order asymptotic value of $i\left(\sigma_{s}^{m} /\left\|\sigma_{s}^{m}\right\|_{L^{2}}\right)$ on $\bar{\tau}$ as $s \rightarrow \infty$ is determined by

$$
i\left(\frac{\sigma_{s}^{m}\left(\operatorname{det} G_{s}\right)^{1 / 4}}{\left\|\sigma_{s}^{m}\right\|_{L^{2}}}\right)(\bar{\tau}) \sim 2^{n / 2} \pi^{n / 4} \hat{\bar{\tau}}_{0, m}(m)
$$

That is, in terms of the distributional sections $\delta^{m}, m \in P_{X} \cap \mathbb{Z}^{n}$, described in Section 4.2, formula (4.5),

$$
\lim _{s \rightarrow \infty} \frac{\sigma_{s}^{m}\left(\operatorname{det} G_{s}\right)^{1 / 4}}{\left\|\sigma_{s}^{m}\right\|_{L^{2}}}=2^{n / 2} \pi^{n / 4} \delta^{m} .
$$


Proof. We will first compute the asymptotics of $i\left(\sigma_{s}^{m}\right)(\bar{\tau})$, and then simply divide by the results of the previous lemma to obtain the desired expressions. To this end, compute first in the open orbit. From (4.6) we have

$$
\begin{aligned}
i\left(\sigma_{s}^{m}\right)(\bar{\tau}) & =\frac{1}{(2 \pi)^{n}} \int_{\check{X}} w^{m} \mathrm{e}^{-h_{0}^{I_{s}}}\|d Z\|_{K}^{1 / 2} \bar{\tau}_{0} \frac{\omega^{n}}{n !} \\
& =\int_{\check{P}_{X}} \mathrm{e}^{m \cdot y_{s}-\left(x \cdot y_{s}-g_{0}-s \psi\right)}\left(\operatorname{det} G_{s}\right)^{1 / 4}\left(\frac{1}{(2 \pi)^{n}} \int_{\mu^{-1}(x)} \mathrm{e}^{\mathrm{i} m \cdot \theta} \bar{\tau}_{0} d \theta\right) d x \\
& =\int_{\check{P}_{X}} \mathrm{e}^{-s((x-m) \cdot \partial \psi / \partial x-\psi)} \mathrm{e}^{-\left((x-m) \cdot y_{0}-g_{0}\right)}\left(\operatorname{det} G_{s}\right)^{1 / 4} \hat{\bar{\tau}}_{0, m}(x) d x .
\end{aligned}
$$

Let $f_{m}:=(x-m) \cdot \partial \psi / \partial x-\psi$. Then by an argument similar to that in the proof of Lemma 4.12 and by Lemma 4.9, we can use Laplace's approximation with equation (4.8) to obtain as $s \rightarrow \infty$ that

$$
\begin{aligned}
i\left(\sigma_{s}^{m}\right)(\bar{\tau}) & \sim\left(\frac{2 \pi}{s}\right)^{n / 2} \frac{\mathrm{e}^{s \psi(m)+g_{0}(m)} s^{n / 4}(\operatorname{det} \operatorname{Hess} \psi(m))^{1 / 4} \hat{\bar{\tau}}_{0, m}(m)}{\sqrt{\operatorname{det} \operatorname{Hess} \psi(m)}} \\
& =(2 \pi)^{n / 2} s^{-n / 4} \mathrm{e}^{g_{s}(m)}\left(\operatorname{det} \operatorname{Hess}_{m} \psi\right)^{-1 / 4} \hat{\bar{\tau}}_{0, m}(m) .
\end{aligned}
$$

Using Lemma 4.12, we have

$$
\frac{\left(\operatorname{det} G_{s}\right)^{1 / 4}}{\left\|\sigma_{s}^{m}\right\|_{L^{2}}} \sim s^{n / 4}(\operatorname{det} \operatorname{Hess} \psi(m))^{1 / 4} \pi^{-n / 4} \mathrm{e}^{-g_{s}(m)}, \quad s \rightarrow \infty
$$

from which we see that as $s \rightarrow \infty$

$$
\begin{aligned}
i\left(\frac{\sigma_{s}^{m}\left(\operatorname{det} G_{s}\right)^{1 / 4}}{\left\|\sigma_{s}^{m}\right\|_{L^{2}}}\right)(\bar{\tau}) \sim & (2 \pi)^{n / 2} s^{-n / 4} \mathrm{e}^{g_{s}(m)}(\operatorname{det} \operatorname{Hess} \psi(m))^{-1 / 4} \hat{\bar{\tau}}_{0, m}(m) \\
& \times s^{n / 4}(\operatorname{det} \operatorname{Hess} \psi(m))^{1 / 4} \pi^{-n / 4} \mathrm{e}^{-g_{s}(m)} \\
= & 2^{n / 2} \pi^{n / 4} \hat{\bar{\tau}}_{0, m}(m)
\end{aligned}
$$

as desired.

Observe that the asymptotics of the normalized sections $\sigma_{s}^{m} /\left\|\sigma_{s}^{m}\right\|_{L^{2}}$ described by Theorem 4.13 contain the additional term $\left(\operatorname{det} G_{s}\right)^{1 / 4}$. This extra factor is better understood in the context of the degeneration of $\mathcal{H}_{I_{s}}^{Q}$ which we now study.

To have the spaces $\Gamma\left(\sqrt{\left|K_{s}\right|}\right)$, for all $s \geq 0$, as subspaces of a given fixed vector space, we consider $\alpha \in \Gamma\left(\sqrt{\left|K_{s}\right|}\right)(U)$, for an open set $U \subset X$, as a map $\alpha: \mathcal{X}(U)^{n} \rightarrow C(U)$, where $\mathcal{X}(U)$ is the space of smooth complex vector fields on $U$ and $C(U)$ is the space of continuous complex valued functions on $U$. Then, we define

$$
\lim _{s \rightarrow \infty} \alpha_{s}=\beta \Leftrightarrow \lim _{s \rightarrow \infty} \alpha_{s}\left(X_{1}, \ldots, X_{n}\right)=\beta\left(X_{1}, \ldots, X_{n}\right),
$$


for all $X_{1}, \ldots X_{n} \in \mathcal{X}(U)$, where on the right-hand side we consider pointwise convergence in $C(U)$. An $n$-form $\beta \in \Omega^{n}(U) \otimes \mathbb{C}$ then also defines a map $\sqrt{|\beta|}: \mathcal{X}(U)^{n} \rightarrow C(U)$, given by $\sqrt{|\beta|}\left(X_{1}, \ldots, X_{n}\right)=\mid \beta\left(X_{1}, \ldots, X_{n}\right)^{\frac{1}{2}}$, for $X_{1}, \ldots, X_{n} \in \mathcal{X}(U)$. Consider now the global $n$-form $d X=d x^{1} \wedge \cdots \wedge d x^{n}$, vanishing on $\cup_{i=1}^{r} D_{i}$, and the corresponding map $\sqrt{|d X|}$.

Lemma 4.14. In the sense defined above,

$$
\lim _{s \rightarrow \infty} \frac{\mu_{I_{s}}}{\left(\operatorname{det} G_{s}\right)^{1 / 4}}=\sqrt{|d X|} .
$$

Proof. On the open orbit, using Lemma 2.12, we have

$$
\frac{\mu_{I_{s}}}{\left(\operatorname{det} G_{s}\right)^{1 / 4}}=\frac{\sqrt{\left|d Z_{s}\right|}}{\sqrt{\operatorname{det} G_{s}}} .
$$

But $d z=G d x+\mathrm{i} d \theta$, which implies $d Z_{s} \sim s^{n} \operatorname{det}\left(\operatorname{Hess}_{x} \psi\right) d X$, and $\operatorname{det} G_{s} \sim$ $s^{n} \operatorname{det}\left(\operatorname{Hess}_{x} \psi\right)$, so

$$
\lim _{s \rightarrow \infty} \frac{\sqrt{\left|d Z_{s}\right|}}{\sqrt{\operatorname{det} G_{s}}}=\lim _{s \rightarrow \infty} \frac{s^{n / 2} \sqrt{\operatorname{det}\left(\operatorname{Hess}_{x} \psi\right)} \sqrt{|d X|}}{s^{n / 2} \sqrt{\operatorname{det}\left(\operatorname{Hess}_{x} \psi\right)}}
$$

as desired. Note that on $\cup_{i=1}^{r} D_{i}$ both sides vanish.

We are now ready to explain the factor of $(\operatorname{det} G)^{1 / 4}$ which appears in Theorem 4.13. As we see, the same term appears in the denominator in Lemma 4.14. Recall the orthogonal basis of $\mathcal{H}_{I_{s}}^{Q}$ given by $\left\{\hat{\sigma}_{s}^{m}=\sigma_{s}^{m} \otimes\right.$ $\left.\mu_{I_{s}}\right\}_{m \in P_{L} \cap \mathbb{Z}^{n}}$, and also that

$$
\left\|\hat{\sigma}_{s}^{m}\right\|_{L^{2}}=\left\|\sigma^{m}\right\|_{L^{2}}
$$

since the Hermitian structure on $\sqrt{\left|K_{I_{s}}\right|}$ gives $\left\|\mu_{I_{s}}\right\|=1$. Combining Lemma 4.14 with Theorem 4.13, we obtain the following theorem.

\section{Theorem 4.15.}

$$
\lim _{s \rightarrow \infty} \frac{\hat{\sigma}_{s}^{m}}{\left\|\hat{\sigma}_{s}^{m}\right\|_{L^{2}}}=\lim _{s \rightarrow \infty} \frac{\sigma_{s}^{m}}{\left\|\sigma_{s}^{m}\right\|_{L^{2}}} \otimes \mu_{I_{s}}=2^{n / 2} \pi^{n / 4} \delta^{m} \otimes \sqrt{|d X|},
$$

in the sense that

$$
\lim _{s \rightarrow \infty} \frac{i\left(\sigma_{s}^{m}\right) \otimes \mu_{I_{s}}}{\left\|\sigma_{s}^{m}\right\|_{L^{2}}}\left(\tau ; X_{1}, \ldots, X_{n}\right)=2^{n / 2} \pi^{n / 4} \delta^{m}(\tau)\left|d X\left(X_{1}, \ldots, X_{n}\right)\right|^{\frac{1}{2}},
$$

for all test sections $\tau \in \Gamma(\bar{L})$ and smooth complex vector fields $X_{1}, \ldots, X_{n} \in$ $\mathcal{X}(X)$.

Remark 4.16. This Theorem justifies the definition of a natural inner product in $\mathcal{H}_{\mathbb{R}}^{Q}$ defined by declaring $\left\{2^{n / 2} \pi^{n / 4} \delta^{m} \otimes \sqrt{|d X|}\right\}_{m \in P_{X} \cap \mathbb{Z}^{n}}$ to be an orthonormal basis. 
We note that the results of Theorem 4.15 are also valid when $X$ is not compact provided that the growth of $\psi$ at infinity is appropriately controlled, so that the function on $\check{P}_{X}$

$$
\mathrm{e}^{-c \int_{0}^{1} t(x-m) \cdot G_{s}(m+t(x-m)) \cdot(x-m) d t}\left(\operatorname{det} G_{s}\right)^{\frac{1}{2}}
$$

is bounded for some sufficiently small $c>0$. This ensures the existence of the $L^{2}$-norms in question and also that one can still apply the Laplace approximation to obtain the convergence to Dirac delta distributions.

\section{The BKS pairing}

5.1. The BKS half-form pairing. Let $K_{I}$ and $K_{J}$ be the canonical bundles on $X$ associated to two toric Kähler complex structures $I$ and $J$. One may define a non-degenerate sesquilinear pairing $\Gamma\left(K_{I}\right) \times \Gamma\left(K_{J}\right) \rightarrow C^{\infty}(M)$ by comparison with the Liouville form. Specifically, for sections $\alpha \in \Gamma\left(K_{I}\right)$ and $\beta \in \Gamma\left(K_{J}\right)$, define the pairing of $\alpha$ and $\beta$ to be the function

$$
\langle\alpha, \beta\rangle:=\frac{\alpha \wedge \bar{\beta}}{(2 \mathrm{i})^{n}(-1)^{\frac{n(n+1)}{2}} \omega^{n} / n !} .
$$

Suppose for the moment that $K_{I}$ and $K_{J}$ admit square roots. Then the above pairing induces a sesquilinear pairing $\Gamma\left(\sqrt{K_{I}}\right) \times \Gamma\left(\sqrt{K_{J}}\right) \rightarrow$ $C^{\infty}(M)$ via

$$
\langle\mu, \nu\rangle:=\sqrt{\frac{\mu^{2} \wedge \bar{\nu}^{2}}{(2 \mathrm{i})^{n}(-1)^{\frac{n(n+1)}{2}} \omega^{n} / n !}},
$$

for sections $\mu \in \Gamma\left(\sqrt{K_{I}}\right)$ and $\nu \in \Gamma\left(\sqrt{K_{J}}\right)$. Note that when $I=J$, the half-form pairing above reduces to the canonical Hermitian structure (3.3) on $\sqrt{K_{I}}$. Moreover, this pairing of half-forms induces a pairing on the halfform corrected prequantizations of $X$, which is known as the BKS pairing:

$$
\langle s \otimes \mu, t \otimes \nu\rangle_{\mathrm{BKS}}:=\int_{X} h^{\ell}(s, t)\langle\mu, \nu\rangle \frac{\omega^{n}}{n !} .
$$

Let $\ell, L$ be the line bundles over $X$ as in Section 3 . The fact that $\ell \otimes \sqrt{K} \cong$ $L \otimes \sqrt{|K|}$ will motivate the definition of the BKS pairing even in the case when $\sqrt{K}$ does not exist.

Let us examine the half-form pairing on $X$ in a little more detail. A short computation shows that on the open orbit

$$
\left\langle d Z_{I}, d Z_{J}\right\rangle=\operatorname{det}\left(\frac{G_{I}+G_{J}}{2}\right)>0,
$$

since both $G_{I}$ and $G_{J}$ are positive definite. Note that although in general one expects $\left\langle d Z_{I}, d Z_{J}\right\rangle$ to be complex, in the toric case it turns out to be real (and positive) since the unitarization of $K_{I}$ is equal to the unitarization of $K_{J}$, whence the phases do not contribute to the pairing. To put it in another 
way, the pairing of $K_{I}$ and $K_{J}$ is entirely captured by the modulus bundles $\left|K_{I}\right|$ and $\left|K_{J}\right|$. Consequently, we define a pairing between sections of $\sqrt{\left|K_{I}\right|}$ and $\sqrt{\left|K_{J}\right|}$ by

$$
\left\langle\mu_{I}, \mu_{J}\right\rangle:=\frac{\left\langle d Z_{I}, d Z_{J}\right\rangle^{\frac{1}{2}}}{\left\|d Z_{I}\right\|_{K_{I}}^{\frac{1}{2}}\left\|d Z_{J}\right\|_{K_{J}}^{\frac{1}{2}}},
$$

where $\mu_{I}, \mu_{J}$ are defined in (3.4). Then, in the general case when $\sqrt{K}$ may not exist, we define a BKS pairing by

$$
\left\langle\hat{\sigma}_{I}, \hat{\sigma}_{J}\right\rangle:=\frac{1}{(2 \pi)^{n}} \int_{X} h^{L}\left(\sigma_{I}, \sigma_{J}\right)\left\langle\mu_{I}, \mu_{J}\right\rangle \frac{\omega^{n}}{n !},
$$

where $\hat{\sigma}_{I}=\sigma_{I} \otimes \mu_{I} \in \mathcal{H}_{I}^{Q}, \hat{\sigma}_{J}=\sigma_{J} \otimes \mu_{J} \in \mathcal{H}_{J}^{Q}$.

This pairing coincides with the inner product (3.8) in $\mathcal{H}_{I}^{Q}$ when $I=J$. From (5.2), we also see that it coincides with the standard BKS pairing in the case when $\sqrt{K}$ exists.

Let $h_{m}^{I}=(x-m) \frac{\partial g_{I}}{\partial x}-g_{I}$, where $g_{I}$ is the symplectic potential defining the complex structure $I$. From Theorem 3.6 and (5.1) it is straightforward to obtain

Proposition 5.1. In the orthogonal basis $\left\{\hat{\sigma}_{I}^{m}\right\}_{m \in P_{L} \cap \mathbb{Z}^{n}}$ for $\mathcal{H}_{I}^{Q}$ and $\left\{\hat{\sigma}_{J}^{m}\right\}_{m \in P_{L} \cap \mathbb{Z}^{n}}$ for $\mathcal{H}_{J}^{Q}$, we have

$$
\left\langle\hat{\sigma}_{I}^{m}, \hat{\sigma}_{J}^{m^{\prime}}\right\rangle=\delta_{m m^{\prime}} \int_{P_{X}} \mathrm{e}^{-h_{m}^{I}-h_{m}^{J}} \sqrt{\operatorname{det}\left(\frac{G_{I}+G_{J}}{2}\right)} d x,
$$

for $m, m^{\prime} \in P_{L} \cap \mathbb{Z}^{n}$.

5.2. Unitarity. Let $I_{s}$ denote the "simple" family of complex structures on $X$ associated to the symplectic potentials $g_{s}=g_{P}+\varphi+s \psi$ for $s \in[0, \infty)$. (Recall that we could have more general deformations of $I_{0}$ defined by $\psi(s)$.) We can consider the BKS pairing for two values $s, s^{\prime}$ in the same simple family. As we will see, even for these simple families, the BKS pairing is not unitary.

From Proposition 5.1, we see that $I$ - and $J$-holomorphic sections associated with different integral points are orthogonal. In the following, we will therefore consider only one "Fourier" sector at a time, that is, a onedimensional subspace of the quantization space. Let $m \in P_{L} \cap \mathbb{Z}^{n}$, and consider the corresponding monomial section $\hat{\sigma}_{s}^{m} \in \mathcal{H}_{I_{s}}^{Q}$.

Since the BKS pairing $\left\langle\hat{\sigma}_{s}^{m}, \hat{\sigma}_{s^{\prime}}^{m}\right\rangle_{\mathrm{BKS}}$ is real and positive (the integrand is positive), the unitarity of the BKS pairing map for the complex structures $s, s^{\prime}$ is equivalent to

$$
\left\langle\hat{\sigma}_{s}^{m}, \hat{\sigma}_{s^{\prime}}^{m}\right\rangle_{\mathrm{BKS}}=\left\|\hat{\sigma}_{s}^{m}\right\|_{L^{2}} \cdot\left\|\hat{\sigma}_{s^{\prime}}^{m}\right\|_{L^{2}} .
$$


For our choice of monomial sections, writing out the integral in Proposition 5.1 shows that for some function $\alpha \in C^{\infty}\left(\mathbb{R}_{\geq 0}\right)$,

$$
\left\langle\hat{\sigma}_{s}^{m}, \hat{\sigma}_{s^{\prime}}^{m}\right\rangle_{\mathrm{BKS}}=\alpha\left(s+s^{\prime}\right)>0 \text {. }
$$

Unitarity then implies $\alpha\left(s+s^{\prime}\right)=\sqrt{\alpha(2 s) \alpha\left(2 s^{\prime}\right)}$. Putting $\alpha=\mathrm{e}^{f}$, we get

$$
f\left(\frac{s+s^{\prime}}{2}\right)=\frac{f(s)+f\left(s^{\prime}\right)}{2}
$$

and differentiating in $s$ we obtain

$$
\frac{1}{2} f^{\prime}\left(\frac{s+s^{\prime}}{2}\right)=\frac{1}{2} f^{\prime}(s)
$$

Therefore,

Lemma 5.2. The BKS pairing between the $I_{s^{-}}$and $I_{s^{\prime}}$-holomorphic quantizations of $X$ is unitary if and only if for each $m \in P_{L} \cap \mathbb{Z}^{n}$ and for each $s \geq 0$,

$$
\left\|\hat{\sigma}_{s}^{m}\right\|_{L^{2}}=\left\|\hat{\sigma}_{0}^{m}\right\|_{L^{2}} \mathrm{e}^{s b}
$$

for some constant $b$.

Comparing with Lemma 4.12, we see that if (5.6) holds, then we have $b=-2 \psi(m)$ and $\left\|\hat{\sigma}_{0}^{m}\right\|_{L^{2}}=\pi^{n / 2} \mathrm{e}^{2 g_{0}(m)}$. Moreover, replacing $\psi$ by $\psi+$ const does not change the complex structure. Hence, we can assume that $\psi(m)=0$ so that the BKS pairing is unitary if and only if $\left\|\hat{\sigma}_{s}^{m}\right\|_{L^{2}}=\left\|\hat{\sigma}_{0}^{m}\right\|_{L^{2}}$, that is, that the $L^{2}$-norm of $\hat{\sigma}_{s}^{m}$ is independent of $s$.

By the argument above, the following theorem implies that the BKS pairing is not unitary along the simple family $I_{s}, s \geq 0$.

Theorem 5.3. For sufficiently large s,

$$
\frac{d}{d s}\left\|\hat{\sigma}_{s}^{m}\right\|_{L^{2}}^{2} \neq 0
$$

In particular, $\left\|\hat{\sigma}_{s}^{m}\right\|_{L^{2}}$ is not constant, whence the BKS pairing is not unitary.

Proof. We have, using the identity $\left.\frac{d}{d s}\right|_{s=0} \operatorname{det}(A+s X)=\operatorname{det}(A) \operatorname{tr}\left(A^{-1} X\right)$,

$$
\begin{aligned}
\frac{d}{d s}\left\|\hat{\sigma}_{s}^{m}\right\|_{L^{2}}^{2}= & \int_{P_{X}}\left(2\left(\psi-(x-m) \cdot \frac{\partial \psi}{\partial x}\right)\right. \\
& \left.+\frac{1}{2} \operatorname{tr}\left(G_{s}^{-1} \operatorname{Hess} \psi\right)\right) \mathrm{e}^{-2 h_{m}^{I_{s}}} \sqrt{\operatorname{det} G_{s}} d x .
\end{aligned}
$$

Without loss of generality, assume that $\psi$ is scaled so that $\psi(m)=0$. Then by Lemma $4.9,\left(\psi-(x-m) \cdot \frac{\partial \psi}{\partial x}\right)$ is strictly negative, so that for large $s$ the integrand is also strictly negative, since $\operatorname{tr}\left(G_{s}^{-1}\right.$ Hess $\left.\psi\right)$ converges pointwise to zero as $s \rightarrow \infty$, which implies that $\frac{d}{d s}\left\|\hat{\sigma}_{s}^{m}\right\|_{L^{2}}^{2}$ is not equal to zero. 
5.3. Quantum connection. Let $\mathcal{I}$ be the set of compatible toric complex structures on $X$. This naturally corresponds to the space of allowed Guillemin-Abreu symplectic potentials modulo additive constants, since two symplectic potentials define the same holomorphic coordinates iff they differ by an additive constant. Note that if $g_{I}$ is a symplectic potential defining the complex structure $I$, then $g_{I}+s \psi$ where $s \in \mathbb{R}, \psi \in C^{\infty}\left(P_{X}\right)$ will also be an allowed symplectic potential provided that $|s|$ is sufficiently small. Therefore, we can regard $\mathcal{I}$ as an open subset of the affine space $g_{P_{X}}+\left(C^{\infty}\left(P_{X}\right)\right) / \mathbb{R}$. Fix a point $p \in \check{P}_{X}$. In the following we will assume that for each complex structure $I \in \mathcal{I}$ a symplectic potential $g_{I}$ was chosen such that $g_{I}(p)-g_{P_{X}}(p)=0$.

One can define a vector bundle over $\mathcal{I}, \mathcal{H}^{Q} \rightarrow \mathcal{I}$, with fiber $\mathcal{H}_{I}^{Q}$. The bundle $\mathcal{H}^{Q}$ is the natural setting for studying the dependence of Kähler quantization on the choice of complex structure.

As mentioned in the introduction, Axelrod, Della Pietra and Witten in [APW91] (see also the related work [Hit90] of Hitchin), for the case where $\mathcal{I}$ is the space of linear complex structures on $\mathbb{R}^{2 n}$ which are compatible with the standard symplectic form, but without including the half-form correction, introduced a natural unitary connection, which is called the quantum connection, on the analogue of the quantum bundle $\mathcal{H}^{Q}$. This quantum connection is defined to be the projection of the trivial connection in $\mathcal{I} \times \Gamma(\ell)$ to $\mathcal{H}^{Q}$.

In our case, the BKS pairing induces a connection, $\nabla^{Q}$, on $\mathcal{H}^{Q} \rightarrow \mathcal{I}$ as follows. Recall that different Fourier modes are orthogonal with respect to the BKS pairing, so that we can consider each Fourier mode labeled by $m \in P_{L} \cap \mathbb{Z}^{n}$ separately. Consider the monomial sections $\hat{\sigma}_{I}^{m} \in \mathcal{H}_{I}^{q}, I \in \mathcal{I}$, $m \in P_{L} \cap \mathbb{Z}^{n}$. Define $\nabla_{\psi}^{Q}$ by

$$
\left\langle\nabla_{\psi}^{Q} \hat{\sigma}_{I}^{m}, \hat{\sigma}_{I}^{m}\right\rangle=\left.\frac{d}{d s}\right|_{s=0}\left\langle\hat{\sigma}_{I+s \psi}^{m}, \hat{\sigma}_{I}^{m}\right\rangle,
$$

where $\psi \in C^{\infty}\left(P_{X}\right)$ with $\psi(p)=0$ and where $I+s \psi$ denotes the complex structure defined by the symplectic potential $g_{I}+s \psi$ for $|s|$ sufficiently small. Note that, by construction, $\nabla^{Q}$ is unitary.

A final implication of the fact that all of the complex structures arising from points in $\mathcal{I}$ have the same "phases" (that is, of Lemma 2.9) and of the consequent reality of the BKS pairing is the following theorem.

Theorem 5.4. The global frame for $\mathcal{H}^{Q} \rightarrow \mathcal{I}$ given by $\left\{\hat{\sigma}_{I}^{m} /\left\|\hat{\sigma}_{I}^{m}\right\|\right\}_{m \in P_{L} \cap \mathbb{Z}^{n}}$ is horizontal with respect to $\nabla^{Q}$. Therefore, the connection $\nabla^{Q}$ is flat.

Proof. We have, from the reality of the BKS pairing, for each Fourier mode $m \in P_{L} \cap \mathbb{Z}^{n}$ and for any $\psi \in C^{\infty}\left(P_{X}\right)$ with $\psi(p)=0$,

$$
0=\left.\frac{d}{d s}\right|_{s=0}\left\langle\frac{\hat{\sigma}_{I+s \psi}^{m}}{\left\|\hat{\sigma}_{I+s \psi}^{m}\right\|}, \frac{\hat{\sigma}_{I+s \psi}^{m}}{\left\|\hat{\sigma}_{I+s \psi}^{m}\right\|}\right\rangle=2\left\langle\nabla_{\psi}^{Q} \frac{\hat{\sigma}_{I}^{m}}{\left\|\hat{\sigma}_{I}^{m}\right\|}, \frac{\hat{\sigma}_{I}^{m}}{\left\|\hat{\sigma}_{I}^{m}\right\|}\right\rangle,
$$

when $\nabla_{\psi}^{Q} \frac{\hat{\sigma}_{I}^{m}}{\left\|\hat{\sigma}_{I}^{m}\right\|}=0$. 


\section{Appendix: The square root of $K$}

For toric varieties, the following proposition explains how the existence of $\sqrt{K}$ depends on the combinatorics of the fan $\Sigma$ associated to $X$. Note also that, in general, if a square root of the canonical bundle exists then there may be many choices of square root, and they are parameterized by $H^{1}\left(X, \mathbb{Z}_{2}\right)$. For toric varieties, $H^{1}\left(X, \mathbb{Z}_{2}\right)=\{0\}$, and so if it exists, $\sqrt{K}$ is unique.

Let $\left\{\nu_{j}\right\}_{j=1, \ldots, r}$ be primitive generators of the one-dimensional cones in the fan $\Sigma$. Let $L$ be a holomorphic line bundle on $X$. The divisor of a torus-invariant meromorphic section of $L$ on any holomorphic vertex chart $U_{v}$ determines the section up to multiplicative constant. Indeed, a torusinvariant principal divisor is of the form

$$
\sum_{i=1}^{r}\left\langle\alpha, \nu_{j}\right\rangle D_{j},
$$

for $\alpha \in \mathbb{R}^{n}$. If, say, $\nu_{1}, \ldots, \nu_{n}$ are the generators associated to $U_{v}$, which form a basis of $\mathbb{Z}^{n}$, then the restriction of the principal divisor to $U_{v}$ determines it completely since $\alpha$ is fixed by its inner products with $\nu_{1}, \ldots, \nu_{n}$. Such a line bundle has a system of meromorphic locally trivializing sections $\left\{\mathbf{1}_{v}^{L}\right\}$ on the holomorphic vertex charts, as in Section 2.2.3, defined uniquely (up to constants) by the property that $\operatorname{div}\left(\mathbf{1}_{v}^{L}\right)_{U_{v}}=0$.

If $c_{1}(X)$ is even then $\sqrt{K}$ exists and it has a system of holomorphic trivializations on the holomorphic vertex charts given by $\left\{\left(U_{v}, \mathbf{1}_{v}\right)\right\}$, where $\mathbf{1}_{v}:=\mathbf{1}_{v}^{\sqrt{K}}$. Then, $\left\{\left(U_{v}, \mathbf{1}_{v} \otimes \mathbf{1}_{v}\right)\right\}$ gives a system of trivializing sections for $K$ and we have, up to an irrelevant constant, $\mathbf{1}_{v} \otimes \mathbf{1}_{v}=d W_{v}$. Therefore, the sections $d W_{v}$ have even divisors and we can write $\mathbf{1}_{v}=\sqrt{d W_{v}}$ where $\left\{\left(U_{v}, \sqrt{d W_{v}}\right)\right\}$ is a system of holomorphic trivializing sections on the holomorphic vertex charts for $\sqrt{K}$. We therefore have

Proposition 6.1. If $c_{1}(X)$ is even, then the sections $d W_{v}$ have even divisors and $\left\{\left(U_{v}, \sqrt{d W_{v}}\right)\right\}$ is a system of holomorphic trivializations of $\sqrt{K}$.

As a consequence, we obtain the following useful criterion for the existence of $\sqrt{K}$. For a vertex $v$, let us call vertex basis associated to $v$ to a basis of $\mathbb{Z}^{n}$ given by the primitive generators of the one-dimensional cones defining $v$.

Proposition 6.2. $K$ admits a square root if and only if for each 1-dimensional cone $F$ in $\Sigma$ the sum of the coordinates of the primitive generator $\nu_{F}$ expressed in any one of the vertex basis is odd.

Proof. We have seen that the existence of $\sqrt{K}$ is equivalent to having a system of trivializing sections $\left\{\sqrt{d W_{v}}\right\}$ where the $d W_{v}$ all have even divisors. Choose a vertex $v$ and let $\nu_{1}, \ldots, \nu_{n}$ be a vertex basis for $v$. Then, using 
Lemma 2.10 and the fact that $d Z_{v}$ is a constant multiple of $d Z$,

$$
\operatorname{div}\left(d W_{v}\right)=\operatorname{div}\left(w_{v}^{\mathbf{1}}\right)+\operatorname{div}\left(d Z_{v}\right)=\sum_{i=n+1}^{r}\left(\sum_{j=1}^{n} \nu_{i}^{j}\right) D_{i}-\sum_{i=n+1}^{r} D_{i},
$$

where $\nu_{i}^{j}$ is the $j$ th coordinate of the vector $\nu_{i}$ in the vertex basis. Therefore, this divisor is even iff $\sum_{j=1}^{n} \nu_{i}^{j}$ is odd for all $i$. Clearly, this happens for any $v$ and the proposition follows.

\section{References}

[Abr03] M. Abreu, Kähler geometry of toric manifolds in symplectic coordinates, Symplectic and contact topology: interactions and perspectives (Toronto, ON/Montreal, QC, 2001), Fields Inst. Commun. 35, Amer. Math. Soc., Providence, RI, 2003, pp. 1-24.

[AGL07] J. Andersen, N. Gammelgaard and M. Lauridsen, Hitchin's connection in halfform quantization, arXiv:0711.3995.

[APW91] S. Axelrod, S. Della Pietra and E. Witten, Geometric quantization of Chern-Simons gauge theory, J. Differ. Geom. 33(3) (1991), 787-902.

[BFMN11] T. Baier, C. Florentino, J.M. Mourão and J.P. Nunes, Toric Kähler metrics seen from infinity, quantization and compact tropical amoebas, J. Differ. Geometry, 89 (2011), 411-454.

[BMN10] T. Baier, J.M. Mourão and J.P. Nunes, Quantization of abelian varieties: distributional sections and the transition from Kähler to real polarizations, J. Funct. Anal. 258 (2010), 3388-3412.

[C78] J. Czyz, "On some approach to geometric quantization", in Differential Geometric Methods in Mathematical Physics II, Lecture Notes in Mathematics 676 (1978), 315-328.

[CdS] A. Cannas da Silva, Symplectic toric manifolds, in 'Symplectic Geometry of Integrable Hamiltonian Systems', Birkhäuser series Advanced Courses in Mathematics — CRM Barcelona, Birkhäuser (Springer), 2003.

[CK] D.A.Cox and S. Katz, Mirror symmetry and algebraic geometry, Math. Surveys and Monographs 68, Amer. Math. Soc. 1991.

[CLS11] D.A. Cox, J.B. Little and H. Schenck, Toric varieties, Graduade Studies in Mathematics 124, AMS, 2011.

[D88] T. Delzant, Hamiltoniens périodiques et images convexes de l'application moment, Bull. S.M.F. 116 (1988), 315-339.

[DP09] J. Duistermaat and A. Pelayo, Reduced phase space and toric variety coordinatizations of Delzant spaces, Math. Proc. Cambridge Philosophical Society 146 (2009), 695.

[F] W. Fulton, Introduction to toric varieties, Annals of Math. Studies 131, Princeton University Press, Princeton, New Jersey, 1993.

[FMMN05] C. Florentino, P. Matias, J.M. Mourão and J.P. Nunes, Geometric quantization, complex structures and the coherent state transform, J. Funct. Anal. 221(2) (2005), 303-322. 
[FMMN06] C. Florentino, P. Matias, J.M. Mourão and J.P. Nunes, On the BKS pairing for Kähler quantizations of the cotangent bundle of a Lie group, J. Funct. Anal. 234(1) (2006), 180-198.

[Gui94] V. Guillemin, Kähler structures on toric varieties, J. Differ. Geom. 40(2) (1994), 285-309.

[GV] I. Gelfand, N. Vilenkin, Generalized functions, Vol. 4. Applications of harmonic analysis, Academic Press, New York 1964.

[Hal02] Brian C. Hall, Geometric quantization and the generalized Segal-Bargmann transform for Lie groups of compact type, Comm. Math. Phys. 226 (2002), 233-268.

[Ham07] M. Hamilton, Locally toric manifolds and singular Bohr-Sommerfeld leaves, Mem. Amer. Math. Soc. 207 (2010), 971.

[Hit90] N.J. Hitchin, Flat connections and geometric quantization, Comm. Math. Phys. 131(2) (1990), 347-380.

[Hör90] L. Hörmander, The analysis of linear partial differential operators I, SpringerVerlag, 1990.

[Huy05] D. Huybrechts, Complex geometry, Universitext, Springer-Verlag, Berlin, 2005.

[KW06] W.D. Kirwin, S. Wu, Geometric quantization, parallel transport and the Fourier transform, Comm. Math. Phys. 266 (2006), 577-594.

[LS10] L. Lempert, R. Szöke, Uniqueness in geometric quantization, arXiv:1004. 4863.

[Mor07] A. Moroianu, Lectures on Kähler geometry, London Mathematical Society Student Texts, 69, Cambridge University Press, Cambridge, 2007.

[Raw79] J.H. Rawnsley, A nonunitary pairing of polarizations for the Kepler problem, Trans. Amer. Math. Soc. 250 (1979), 167-180.

[Ś75] J. Śniatycki, On cohomology groups appearing in geometric quantization, Lecture Notes in Mathematics 570 (1975).

[Wei04] A. Weinstein, The Maslov gerbe, Lett. Math. Phys. 69 (2004), 3-9.

[Woo91] N. Woodhouse, Geometric quantization, Oxford University Press, Oxford, 1991.

[Wu10] S. Wu, Projective flatness in the quantization of bosons and fermions, arXiv: 1008.5333.

Mathematics Institute

University of Cologne

WEYERTAL 86-90

50931 COLOGNE

GERMANY

E-mail address: will.kirwin@gmail.com

Center for Mathematical Analysis

Geometry and Dynamical Systems 


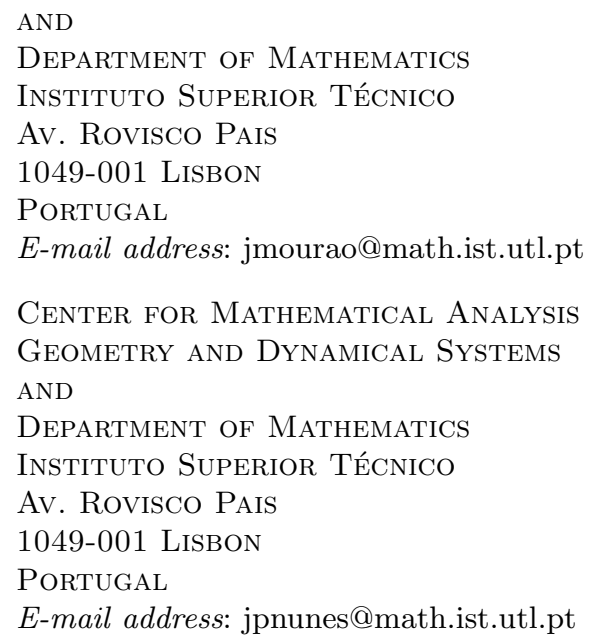

Received 11/28/2011, accepted 11/27/2012

We wish to thank Thomas Baier for extensive discussions on the subject of the paper. We also wish to thank the referee for several helpful comments which helped improving the clarity of the text. The authors are partially supported by the Center for Mathematical Analysis, Geometry and Dynamical Systems, IST, through the Fundação para a Ciência e a Tecnologia (FCT/Portugal). 
